\title{
SIMULATIONS OF ALPHA PARAMETERS IN A TFTR SUPERSHOT WITH HIGH FUSION POWER
}

\author{
BY
}

R.V. BUDNY, M.G. BELL, A.C. JANOS, ET AL.

\author{
JULY 1995
}
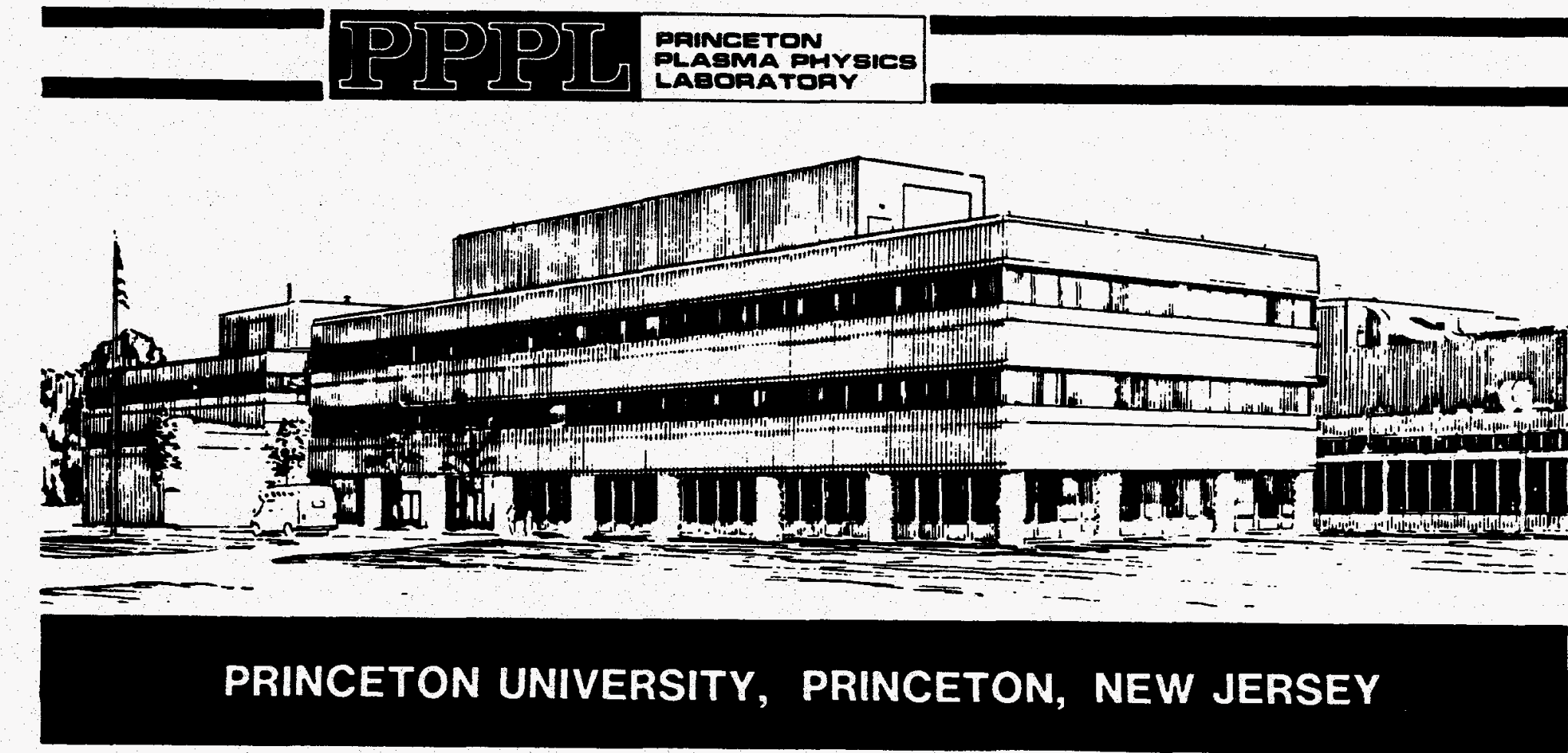


\section{NOTICE}

This report was prepared as an account of work sponsored by an agency of the United States Government. Neither the United States Government nor any agency thereof, nor any of their employees, makes any warranty, express or implied, or assumes any legal liability or responsibility for the accuracy, completeness, or usefulness of any information, apparatus, product, or process disclosed, or represents that its use would not infringe privately owned rights. Reference herein to any specific commercial produce, process, or senvice by trade name, trademark, manufacturer, or otherwise, does not necessarily constitute or imply its endorsement, recommendation, or favoring by the United States Government or any agency thereof. The views and opinions of authors expressed herein do not necessarily state or reflect those of the United States Government or any agency thereof.

\section{NOTICE}

This report has been reproduced from the best available copy.

Available in paper copy and microfiche.

Number of pages in this report: 39

DOE and DOE contractors can obtain copies of this report from:

Office of Scientific and Technical Information

P.O. Box 62

Oak Ridge, TN 37831 ;

(615) $576-8401$.

This report is publicly available from the:

National Technical Information Service

Department of Commerce

5285 Port Royal Road

Springfield, Virginia 22161

(703) $487-4650$ 


\section{DISCLAIMER}

Portions of this document may be illegible in electronic image products. Images are produced from the best available original document. 


\title{
Simulations of Alpha Parameters in a TFTR DT Supershot with High Fusion Power
}

\author{
R.V. Budny, M.G. Bell, A.C. Janos, D.L. Jassby, \\ L.C. Johnson, D. Mansfield, D.C. McCune, M.H. Redl, J.F. Schivell, \\ G. Taylor, T.B. Terpstra, M.C. Zarnstorf, and S. J. Zweben
}

\author{
Princeton University \\ Plasma Physics Laboratory \\ P. O. Box 451, Princeton NJ, 08544
}

May 30, 1995

Abstract

A TFTR supershot with a plasma current of 2.5 MA, neutral beam heating power of 33.7 MW, and a peak DT fusion power of 7.5 MW is studied using the TRANSP plasma analysis code. Simulations of alpha parameters such as the alpha heating, pressure, and distributions in energy and $v_{\text {parallel }} / v$ are given. The effects of toroidal ripple and mixing of the fast alpha particles during the sawteeth observed after the neutral beam injection phase are modeled. The distributions of alpha particles on the

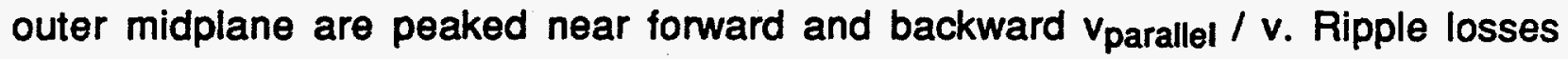
deplete the distributions in the vicinity of $v_{\text {parallel }} / v \approx-0.4$. Sawtooth mixing of fast alpha particles is computed to reduce their central density and broaden their width in energy.

DISTRIBUTION OF THIS DOCUMENT IS UNLIMITED

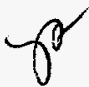

Alpha Parameters, page 1 


\section{Introduction}

Experiments with deuterium-tritium (DT) plasmas were started in TFTR during $1993[1,2,3]$. Several experiments have been dedicated to maximizing the fusion power. In November 1994, DT discharges with neutral beam injection (NBI) heating of $39 \mathrm{MW}$, plasma currents of 2.7 MA, and toroidal fields of 5.6 T achieved fusion power at peak rates up to $10.7 \mathrm{MW}$ and at peak power densities above $2.5 \mathrm{MW} / \mathrm{m}^{3}$.

The previous record was set in May 1994, when DT discharges with NBI heating of $34 \mathrm{MW}$, plasma currents of $2.5 \mathrm{MA}$, and toroidal fields of $5.1 \mathrm{~T}$ achieved fusion power at peak rates up to $9.2 \mathrm{MW}$ [4]. The discharge with the highest peak rate disrupted, but similar discharges with peak rates up to $7.5 \mathrm{MW}$ did not disrupt and had sustained high fusion power production for approximately $0.5 \mathrm{sec}$. Analysis of the highest nondisruptive discharge from May 1994 with a peak rate of $7.5 \mathrm{MW}$ is discussed in this paper.

This discharge has been extensively modeled using the TRANSP timedependent plasma analysis code [5-8]. The modeling provides calculations of profiles of the $D$ and $T$ densities, the hydrogenic isotopic mass, the fusion reactions, fast ion parameters, and the alpha heating. Results are in approximate agreement $(\sim 1 \sigma)$ with available measurements [5].

A detailed prediction for a DT supershot based on a moderate-performance, reproducible D-only supershot was described before the DT campaign [7]. That simulation predicted 5.6 MW of fusion power with a fusion power ratio $Q_{D T} \equiv P_{D T} / P_{N B}$ $=0.23$. This paper updates those simulations using an actual high-performance DT discharge. One of the predictions [7] made before the DT campaign was that the stored energy in DT-NBI supershots would be about $10 \%$ higher than in D-only supershots. This predicted increase is due to changes in the NBI parameters, the effects of alphaelectron heating, and the additional stored energy of the alphas. The observed increase is twice as large [1]. The extra increase is attributed to an intrinsic isotopic scaling of the thermal plasma confinement with isotopic mass $[8,9]$.

Several methods are being used to measure facets of the confined alpha particle distributions in TFTR plasmas. One method uses charge exchange recombination spectroscopy of alphas excited by NBI ions [10]. Another method analyses the energy of neutral alpha particles from double charge exchange in pellet ablation clouds [11]. A third method is designed to analyze gyrotron Thomson scattering from alpha particles 
[12]. These diagnostics measure the alpha energy in different regions of energy and $\lambda \equiv$ $v_{\text {parallel }} / v \equiv \cos$ (pitch angle). This paper gives simulations for the alpha distributions in energy and $\lambda$.

Supershots generally exhibit sawteeth before and after the NBI phase. These sawteeth are accompanied by significant decreases in the central plasma parameters such as $T_{e}$ and $n_{e}$. If the fast ions are mixed during the sawteeth crashes, then the central neutron emission and alpha parameters would be affected. Fast ion mixing is modeled in TRANSP and simulations of this mixing are being used to test empirically whether such mixing occurs.

General plasma conditions for the discharge are discussed in Section 2. The TRANSP modeling typically used for TFTR discharges is discussed in Section 3. Alpha particle parameters are discussed in Section 4. The fast alpha distributions are discussed in Section 5.

\section{Plasma Conditions for 76770}

The neutral beams injected 19.6 MW of T and $14.1 \mathrm{MW}$ of D from 3.5 to $4.3 \mathrm{sec}$. Plasma conditions at the time of peak neutron emission rate $(\approx 4.0 \mathrm{sec})$ are $I_{p}=2.5 \mathrm{MA}$, $B_{T F}=5.1 \mathrm{~T}, R_{0}=2.5 \mathrm{~m}, a=0.9 \mathrm{~m}$, and $q_{\psi}(a)=4$. The $q_{\psi}$ profile was not measured, but $q_{\psi}(0)=0.8$ is typically measured in similar discharges. The total energy confinement time is $0.22 \mathrm{sec}$. This relatively high confinement time resulted from intensive Li pellet injection [13] into the ohmic phases of discharges during this experimental run in order to condition the vessel walls.

Supershots generally exhibit sawteeth during their ohmic phases, and occasionally during the neutral beam phase. Typical behavior with high NBI power is that the pre-NBI sawteeth disappear after one or two crashes during the start of the NBI. After $\approx 0.1 \mathrm{sec}$, sawteeth are generally not observed during the NBI except if the NBI power is low ( $<25 \mathrm{MW}$ ) and the plasma current $\mathrm{I}_{\mathrm{p}}$ is high ( $>2 \mathrm{MA}$ ). They start about = $0.2 \mathrm{sec}$ after the termination of NBI with regularity. Examples of the effects of sawteeth and the $L i$ pellet for 76770 are seen in the $T_{\theta}(0)$ trace in Fig. 1. Empirical evidence for degree to which the beam ions and fusion alpha ions are mixed during sawteeth crashes is not fully established. This mixing is computed to have significant consequences on the energy and pitch angle distributions of the fast ions. 
There is little direct evidence for alpha particle effects on the plasma in supershots. One indirect piece of evidence is that the measured alpha losses are normal in supershots that do not have excessive MHD activity [14]. The measured rates of fast alpha particles collected in detectors at poloidal angles $20^{\circ}[15]$ and $90^{\circ}$ below the midplane for 76770 , normalized to the total neutron emission rate are approximately constant in time.

\section{TRANSP Modeling Capabilities}

The TRANSP plasma analysis code is used to model the time evolutions of plasma parameters. The energy, particle, and magnetic field dynamics are computed. Measured plasma profiles and the location of the last closed flux surface are specified. Three temperatures ( $T_{e}$, hydrogenic ion $T_{i}$, and impurity ion $T_{x}$ ) are modeled. Typically, measurements of the electron temperature (from ECE) and of the temperature and toroidal rotation velocity of the trace carbon impurity (from neutral-beam induced charge-exchange recombination spectroscopy) are used as initial inputs. The hydrogenic temperature $T_{i}$ is computed from the measured $T_{x}$ in TRANSP [6]. This computed central $T_{i}$ is lower than $T_{x}$ by several $k e V$. The difference is largest during the first $\sim 0.2$ sec of the NBI.

Up to five thermal ion species and one impurity species can be modeled simultaneously in TRANSP. For the modeling described here, the thermal species are $H, D, T, \mathrm{He}^{4}, \mathrm{Li}$, and one heavier impurity. The hydrogen and impurity species are nonnegligible components, as discussed in Ref. [5]. The lithium has small relative concentrations during the NBI phase. The helium ash is estimated to have small relative concentrations (peaking at $n_{\theta} / 1000$ in the center).

The equilibrium flux surfaces are calculated by solving the fixed-boundary GradShafranov equations using the total (beam + thermal) pressure. Typically six seconds of the discharge evolution are modeled, starting within 0.5 sec of the discharge initiation and continuing through the neutral beam injection. Modeling the early phase allows a more accurate computation of the magnetic field diffusion and the $q_{\psi}$ profile.

Fast lons - Monte-Carlo techniques [16] are used to compute the deposition of the neutral beams, and the distributions of the beam ion and fusion ions. The beam ions and fusion products are assumed to slow down either classically or with an added anomalous radial diffusivity. The assumptions of classical slowing down and loss have 
been checked in the case of beam ions [17] and DD fusion products [18]. For instance, if a constant radial diffusivity of $\sim 0.05 \mathrm{~m}^{2} / \mathrm{sec}$ is assumed to be superimposed on the classical slowing down of the beam ions, then the TRANSP results for the total neutron rates and the stored energies would differ significantly from the measurements [17]. We extrapolate these results by assuming no anomalous diffusion for the fast alpha particles. The losses of beam ions and alpha particles on orbits that intercept the limiter or wall are computed in TRANSP.

The TRANSP modeling is tested and confirmed by comparing simulations with measurements. Examples of comparisons are the Shafranov shifts, the stored energy, and the neutron emission profile. These tests are interrelated and provide global checks of the modeling. For instance, the Shafranov shifts, which depend on the total pressure, effect the plasma profiles that are used to calculate the plasma and beam pressure. Comparisons are discussed in detail in Ref. [5].

Neutron emission - The measured and simulated total neutron emission rates are shown in Fig. 2-a. The absolute measurement uncertainty is $1 \sigma \approx 7 \%$. These are in good agreement for the first $0.6 \mathrm{sec}$ of NBI. Later the simulated total is systematically higher than the measured value. The neutron emission chordal profiles are measured by a collimator array. The absolute measurement error is $1 \sigma= \pm 15 \%$. Time evolutions of the simulations and measurements are compared in Fig. 2-b. Profiles near the time of peak emission are compared in Fig. 2-c. The simulated profiles are shifted several $\mathrm{cm}$ outward with respect to the measured profiles. Accurate simulations of the central neutron emission rates are desired since the alpha parameters depend sensitively on the central fusion rate. The simulation depends sensitively on the $Z_{\text {eff }}$ of the plasma, which is not known accurately for reasons discussed in Ref. [5].

Fusion Power - The peak neutron emission rate is $S_{n}=2.7 \times 10^{18} / \mathrm{sec}$, so the fusion yield, PDT ( $\equiv S_{n} / 3.6 \times 10^{17}[\mathrm{MW} / \mathrm{sec}$ ) ) is $7.5 \mathrm{MW}$. The calculated fraction of the total from thermonuclear reactions is $25 \%$. The central fusion power density is dominantly thermonuclear, as shown in Fig. 3. The central alpha-electron heating rate is shown for comparison. Its peak occurs after the peak fusion rate. This is discussed in more detail in Section 4.

Ripple - A model for estimating toroidal field ripple effects of fast ions has been included in TRANSP $[19,20]$. The magnitude of the toroidal field ripple is input along with a threshold set as a factor times the Goldston-White-Boozer [21] stochastic ripple threshold. When the turning points of fast ion orbits are in locations where the toroidal field ripple is above this threshold, the ions are ejected from the plasma. The choice of 
the multiplier has been adjusted by comparisons of TRANSP and guiding center code calculated losses.

Sawteeth - The occurrence of sawteeth in TFTR supershots is consistent with a criterion of drift frequency, $\omega_{*}$-stabilization of the collisionless $m=1$ reconnection mode $[22,23]$. Sawteeth may have important implications for alpha parameters due to mixing. TRANSP uses a phenomenological sawtooth model which is invoked at the observed sawtooth crash times if $q_{\psi}(0)$ is less than 1 . The magnetic flux surfaces are shifted using the Kadomtsev helical flux mixing model [24]. The calculated mixing radius is $\approx 30 \%$ larger than the calculated $q_{\psi}=1$ radius.

The amount of current mixing can be adjusted in TRANSP to less than the full current mixing in the standard Kadomtsev model. A weighted average of the unmixed and fully mixed current profiles is imposed after the crash. For the results given here the weighting is $70 \%$ of the unmixed and $30 \%$ of the fully mixed current. These ranges predict $q_{\psi}(0)$ values in approximate agreement with observations. Full $(100 \%)$ current mixing predicts $q_{\psi}(0)$ values which increase after the sawteeth crashes to values that are too high (close to one) for consistency with measurements [25]. The sawtooth prescription in TRANSP generally calculates the $(m, n)=(1,1),(4,3)$, and $(3,2)$ surfaces which are within $\sim 5 \mathrm{~cm}$ of the locations of sawteeth inversions and of coherent $(4,3)$ and $(3,2)$ MHD activity indicated by fast $T_{e}$ measurements.

TRANSP can be run either with or without fast ion mixing. In the case where the fast ions are assumed to mix, the ions are shifted with the flux surfaces conserving helical flux. The poloidal angle for each Monte Carlo ion is randomized and the values of $v_{\text {parallel }}$ and $\mu \equiv v_{\text {perp }} 2 / B$ are conserved through the crash. This prescription alters the energies and $\lambda \equiv v_{\text {parallel }} / v$ of the fast ions and converts some of the passing ions to trapped and vice versa.

\section{Alpha Parameters}

The alpha slowing down and pitch angle scattering are modeled. The slowing down and pitch angle scattering times are considerable longer than the corresponding times for beam ions, as shown in Figs. 4. The confined alphas are calculated to mainly heat electrons. The alpha-electron heating is predicted to be a significant portion of the total election heating in the center. Its profile is compared with the beam-electron and thermal ion-electron heating in Fig. 5. 
The calculated time evolutions of the central fast alpha particle density $n_{\alpha}$, average energy $\left\langle\mathrm{E}_{\alpha}\right\rangle$, toroidal beta $\beta_{\alpha}$, and electron heating power are shown in Figs. 6. During the NBI phase $n_{\alpha}(0)$ increases steadily, while $\left\langle E_{\alpha}(0)>\right.$ decreases. These contrary trends cause $\beta_{\alpha}(0)$ to peak during the NBI. Results are given with and without fast ion mixing. If the fast ions are assumed to mix during the sawtooth crash with the prescription described in the previous section, these central parameters decrease precipitously after the crash. If the ions are not mixed by sawteeth, these central parameters tend to decrease gradually after the termination of NBI. In this case, the alpha heating peaks during the sawteeth crashes and during the pellet (due to the rapid thermalization in the high density pellet cloud).

The fast ion mixing prescription described above has dramatic effects on the fast ion profile, as shown in Fig. 7. The independent variable is the square root of the normalized toroidal flux, $x$. This variable is close in value to the normalized minor radius, r/a.

The ripple model predicts effects on alpha parameters that are more subtle than the sawtooth mixing. Profiles are shown in Figs. 8. The ripple losses of energy for the alphas, D-beam ions, and T-beam ions are $9 \%, 11 \%$, and $13 \%$, respectively. The particle losses are about $1 \%$ higher. The tirst-orbit losses are relatively small $(\approx 3 \%)$ since the plasma current is relatively high (2.5MA).

\section{5 - Alpha Distributions}

Monte Carlo methods are used to track orbits of individual fast ions. The parameters for these are sampled to generate their distribution in the laboratory rest frame,

$$
f_{j}\left(E_{j}, \lambda_{j}, x, t\right) \equiv \frac{d n_{j}}{d V d E_{j} d \lambda_{j}}
$$

The independent variables are the energy $E_{j}$ of the fast species $j, \lambda_{j} \equiv v_{\text {parallel }} / v \equiv$ $\cos$ (pitch angle) with $v_{\text {parallel }}$ the component of $v$ in the direction of the plasma current, and the location in space and time. These are normalized so that their integral over $E_{j}$ and average over $\lambda_{j}$ gives the fast ion spatial density. An example of the flux-surface average is shown in Fig. 8-a. 
These distributions are formed by censusing the fast ions passing through zones in a 2 dimensional slice at fixed toroidal angle. For the results given here, 100 equal energy intervals and 50 equal $\lambda$ intervals are used. The zones are chosen to lie along the outer midplane. The radial spacing of the zones are equal intervals of $x$. The poloidal widths of the zones are indicated in Fig. 9. The distributions are not the same as the orbit averages for the fast ions.

The results given here are from TRANSP runs using 8000 Monte Carlo $D$ and T beam ions, and 8000 fusion alpha ions. The results are averaged over $80 \mathrm{msec}$ at various times during the discharge. These times are selected throughout the NBI phase, before and after the post-NBI sawteeth, and before the post-NBI pellet. The sampling windows are shown in Fig. 1.

The results for the beam ion distributions, $f_{D}$ and $f_{T}$, and for $f_{\alpha}$ are qualitatively similar to the results given in Ref. [7]. More detailed results for $t_{\alpha}$ are given here. First we show the effects of ripple by comparing $f_{\alpha}$ before the first post-NBI sawtooth. The plots in Figs. 10 compare $f_{\alpha}\left(E_{\alpha}, \lambda, x=0.5, t=4.43 s\right)$ calculated with and without the ripple model. The results for both cases show a peaking at positive $\lambda$. This is due to non-zero orbit effects and the $\lambda$ peaking of the beam ions. The alpha distributions have regions with relatively low values near $\lambda \approx-0.4$ and $E_{\alpha} \approx 2.5 \mathrm{MeV}$. This is a consequence of the trapped ions with negative $\lambda$ on the midplane having banana orbits with $x$ increasing away from the midplane, whereas those with positive $\lambda$ have banana orbits with $x$ decreasing away from the midplane. Some of the orbits with increasing $x$ leave the last closed flux surface and can get scraped off. Also the slowing down time decreases with $x$, as shown in Fig. 4-a, so the ions with negative $\lambda$ slow down more rapidly. In the case where the ripple model in turned on (Fig. 10-b), this depletion is more pronounced since the ripple loss is more likely at larger $x$. These effects are seen to a lesser extent in the center $(x \approx 0)$, and to a larger extent at $x>0.5$. At $x=0.7$ these effects are very pronounced, as shown in Figs. 11.

The effects of fast-ion sawooth mixing can be seen in the distributions after the sawtooth crashes. Effects on the energy distribution are shown in Fig. 12. The results are averaged from 4.52 to $4.53 \mathrm{sec}$ and for $-0.1<\lambda<+0.1$. The mixing has the effect of depleting the distribution at $x=0$ and increasing it at $x=0.5$. The mixing and ripple effects on the pitch angle distribution at $x=0.5$ are shown in Figs. 13. Their combined effects cause a trough near $\lambda \approx-0.2$.

Thermal Doppler broadening in $E_{\alpha}$ of $f_{\alpha}$ is not yet included in TRANSP; however, the broadening due to the alphas slowing down and scattering is included. The 
broadening in the center $(x=0)$ is shown in Figs. 14. During the first $0.5 \mathrm{sec}$ of $N B I$ the distribution is inverted. Later it develops into a slowing down distribution. Both ripple and sawtooth mixing effects are included in this result.

\section{6 - Summary}

TRANSP simulations of alpha parameters are given for a high performance, nondisruptive TFTR DT-NBI supershot with a peak fusion power of $7.5 \mathrm{MW}$. Effects of ripple loss and sawteeth are modeled. Trapped fast ions are assumed to be ejected from the plasma if their turning points are above a preset factor times the Goldston-White-Boozer threshold. The ripple losses of alpha particles are estimated to be $\approx 10 \%$ for the plasma discussed in this paper. The sawtooth model in TRANSP for the thermal plasma is a modification of the Kadomtsev flux and current mixing model. In TFTR discharges, the amount of current mixing that gives $q_{\psi}$ profiles in approximate agreement with measurements is about $30 \%$. A model for fast ion mixing during the sawtooth crashes is also incorporated in TRANSP. Their guiding centers are assumed to remain on the flux surfaces, and their parallel velocity is assumed to be conserved. Results with and without fast ion mixing during the post-NBI sawteeth are compared. The central alpha density and pressure are predicted to decrease significantly if the fast ions mix during sawtooth.

Monte Carlo methods are used to calculate fast ion distributions. Results are given for the distributions on the outer midplane versus energy and $\lambda=\cos$ (pitch angle). The central beam ion distributions in $\lambda$ are peaked near forward and backward pitch angles. Ripple losses deplete regions with $\lambda \approx-0.2$ and $E \approx 2.5 \mathrm{MeV}$. Sawtooth mixing of the fast alpha particles is predicted to alter the pitch angle distributions.

\section{Acknowledgments}

We wish to thank J. D. Strachan for encouragement and the U. S. Department of Energy for support under the Contract No. DE-AC02-76-CHO-3073. 


\section{References}

[1] R. J. Hawryluk, H. Adler, P. Alling, et al., Phys. Rev. Lett. 72 (1994) 3530.

[2] J. D. Strachan, H. Adler, P. Alling, et al., Phys. Rev. Lett. 72 (1994) 3526.

[3] J. D. Strachan, H. Adler, P. Alling, et al., Plasma Physics and Controlled Fusion 12 (1994) 33.

[4] M. G. Bell, C. W. Barnes, R. V. Budny, et al., IAEA (A-2-1-2).

[5] R. V. Budny, M. G. Bell, R.E. Bell, et al., PPPL-3039.

[6] R. V. Budny, M. G. Bell, H. Biglari, et al., Nucl. Fusion 32 (1992) 429.

[7] R. V. Budny, Nucl. Fusion 34 (1994)-1247.

[8] R. V. Budny, M. G. Bell, D. K. Mansfield, J.D. Strachan, et al., proceedings of the 21 th EPS, Montpellier, (1994) 18B-I, 82.

[9] S.D. Scott, ot al., Phys of Fluids.

[10] G. R. McKay, R. J. Fonck, T. A. Thorson and B. C. Stratton, "Implementation of alpha CHERS diagnostic for DT operation in TFTR", to be published in Rev. of Scientific Instruments; G. R. McKay, et al., submitted to Phys. Rev. Letters.

[11] R. Fisher, J.M. McChesney, A.W. Howold, et al., Rev. of Scientific Instruments 63 (1992), 4499. M. Petrov, R. Budny, et al., submitted to Nucl. Fusion (this workshop).

[12] J. Machuzak, P. P. Woskov, J. Gilmore, et al., "TFTR $60 \mathrm{GHz}$ Alpha Particle Collective Thomson Scattering Diagnostic," to be published in Rev. of Scientific Instruments.

[13] J. A. Snipes, E. S. Marmar, J. L. Terry, et al., Joumal. of Nucl. Materials 196-198 (1992) 686.

[14] S.J. Zweben, et al., "Alpha Particle Loss in TFTR DT experiments", submitted to Nucl. Fusion.

[15] S.J. Zweben, et al., submitted to Nucl. Fusion (this workshop).

[16] R. J. Goldston, D. C. McCune, H. H. Towner, et al., Journal Comp. Physics, 43 (1981) 61.

[17] E. Ruskov, W. Hedibrink, and R.V. Budny, to be published in Nuclear Fusion.

[18] J.D. Strachan, C.W. Bames, R.V. Budny, et al., to be published in Nuclear Fusion. 
[19] M. H. Redi, M. C. Zamstorff, et al., to be published in Nucl. Fusion.

[20] M. H. Redi, R.V. Budny, et al., submitted in Nucl. Fusion (this workshop).

[21] R. J. Goldston, R. B. White, and A. H. Boozer, Phys. Rev. Lett. 47 (1981) 647.

[22] F.M. Levinton, L. Zakharov, S.H. Batha, et al., Phys. Review Letters, 72 (1994) 2895.

[23] L. Zakharov, F. Levinton, S. Migliuolo and B. Rogers, "The Theory of Stabilization of Sawtooth Oscillations in TFTR Supershots", PPPL report 2953 (1994).

[24] B. B. Kadomtsev, Sov. J. Plasma Phys. 1 (1975) 389.

[25] M. Yamada, F. M. Levinton, N. Pomphrey, et al., Phys. Plasmas 1 (1994) 3269. 


\section{Figure Captions}

Fig. 1 Evolutions of the central electron temperature measured from electron cyclotron emission. The sawteeth and pellet effects on $\mathrm{T}_{\theta}(0)$ are indicated. The sampling windows used for averaging the Monte Carlo data used for the fast ion distributions are also indicated.

Fig. 2 Time evolutions of a) the measured and simulated total neutron emissivity and the simulated contributions from beam-thermal, beam-beam and thermonuclear fusion reactions; b) comparisons of the measured and simulated chordal neutron emissivity; and c) profiles near the time of peak neutron emission.

Fig. 3 Time evolutions of the central fusion power densities of the total DT reactions, of the thermonuclear reactions, and of the alpha-electron heating.

Fig. 4 Profiles of the a) slowing down times, and b) pitch angle scattering times of the alpha particles and beam ions at birth.

Fig. 5 Profiles versus major radius of the dominant electron heating terms showing significant central alpha-electron heating.

Fig. 6 Evolutions of central alpha parameters assuming a) fast ion sawtooth mixing, and b) no fast ion sawtooth mixing. The differences in the time evolutions before the post-NBI sawtooth are due primarily to Monte Carlo fluctuations.

Fig. 7 Profiles a) the fast alpha density and b) $q_{\psi}$ after the first post-NBI sawtooth. The independent variable is the square-root of the normalized toroidal flux (approximately r/a). The effect of sawtooth mixing on the alphas is shown. With the mixing prescription generally used for modeling TFTR discharges, the effect on $q_{\psi}$ is negligible.

Fig. 8 Profiles of the a) fast ion density, and b) $\beta_{\alpha}$ near the end of the NBI phase. The effects of ripple loss are indicated.

Fig. 9 Zones used for computing fast ion distributions. The radial intervals are determined by combining adjacent pairs of the 20 flux surfaces computed by TRANSP in solving for the MHD equilibrium. The poloidal angles are equally spaced within each flux surface and the number of poloidal angle increments increases as the flux increases. The zones for the sampling the distributions on the outer midplane are indicated. Pairs of poloidal zones, one below and one above the midplane are combined for the results presented here. 
Fig. 10 Distribution of fast alphas in $E$ and $\lambda$ at the half-radius, just before the first postNBI sawtooth crash. For a) the ripple model is assumed to be negligible. For b) the usual ripple model is used. The distributions are averaged in energy using a width of $0.1 \mathrm{MeV}$.

Fig. 11 Distribution of fast alphas in $E$ and $\lambda$ at $x=0.7$, just before the first post-NBI sawtooth crash. For a) the ripple model is assumed to be negligible. For b) the usual ripple model is used. The distributions are averaged in energy using a width of $0.1 \mathrm{MeV}$.

Fig. 12 Distribution of fast alphas in $x$ with $\lambda$ near 0.0 , and $E_{\alpha}=0.5$ and $1.0 \mathrm{MW}$, just after the first post-NBI sawtooth crash. Two cases are compared: a) the fast-ion sawtooth mixing and the ripple losses are assumed to be negligible; and b) the usual fast ion mixing and ripple models are used.

Fig. 13 Distribution of fast alphas in $\lambda$ at $x=0.5$, just after the first post-NBI sawtooth crash. The results are averaged in $\lambda$ with $\Delta \lambda=0.2$. Three cases are compared: a) the fast-ion sawtooth mixing and the ripple losses are assumed to be negligible; b) the usual fast ion mixing model is used; and c) the usual fast ion mixing and ripple models are used.

Fig. 14 Broadening of the alpha distribution, averaged over $\lambda$ in the center assuming fast ion sawtooth mixing and usual ripple losses. 
Central $\mathrm{T}_{\mathrm{e}}$ and Sawteeth

\section{Typical Evolution in Supershots}

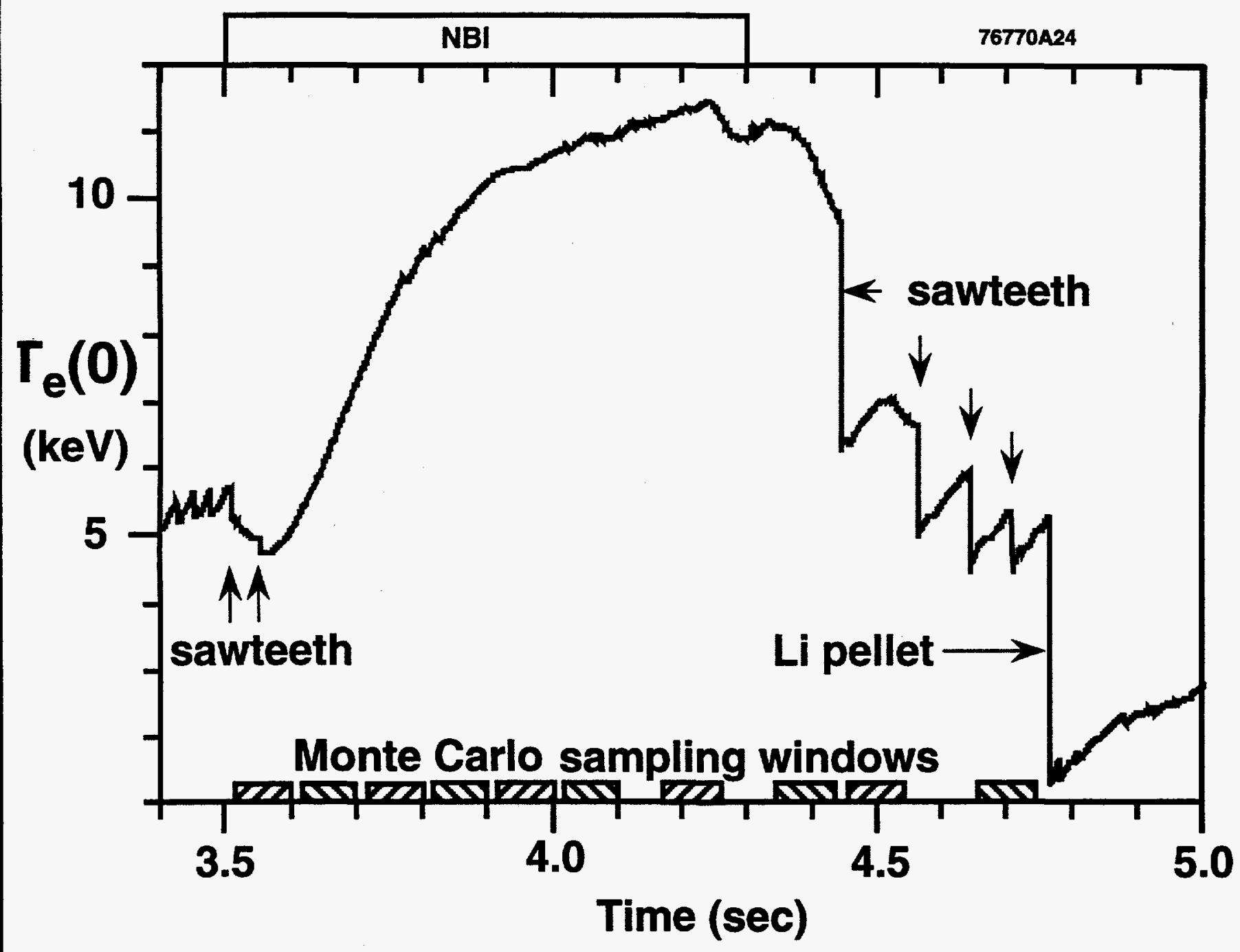

Fig. 1 


\section{Neutron Emission Components DT Supershot}

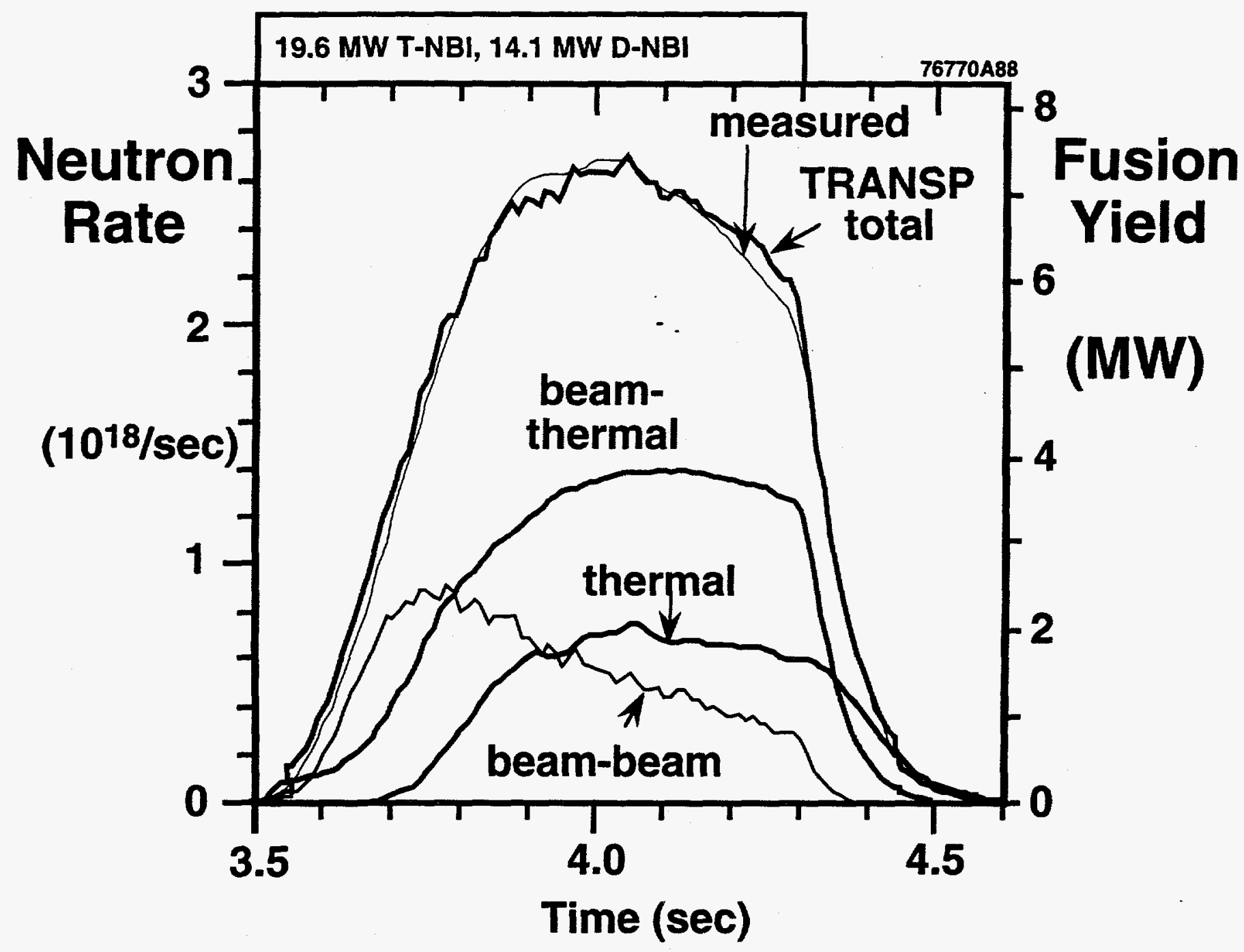

Fig. $2(a)$ 


\section{Neutron Chordal Emission Comparison with measurements}

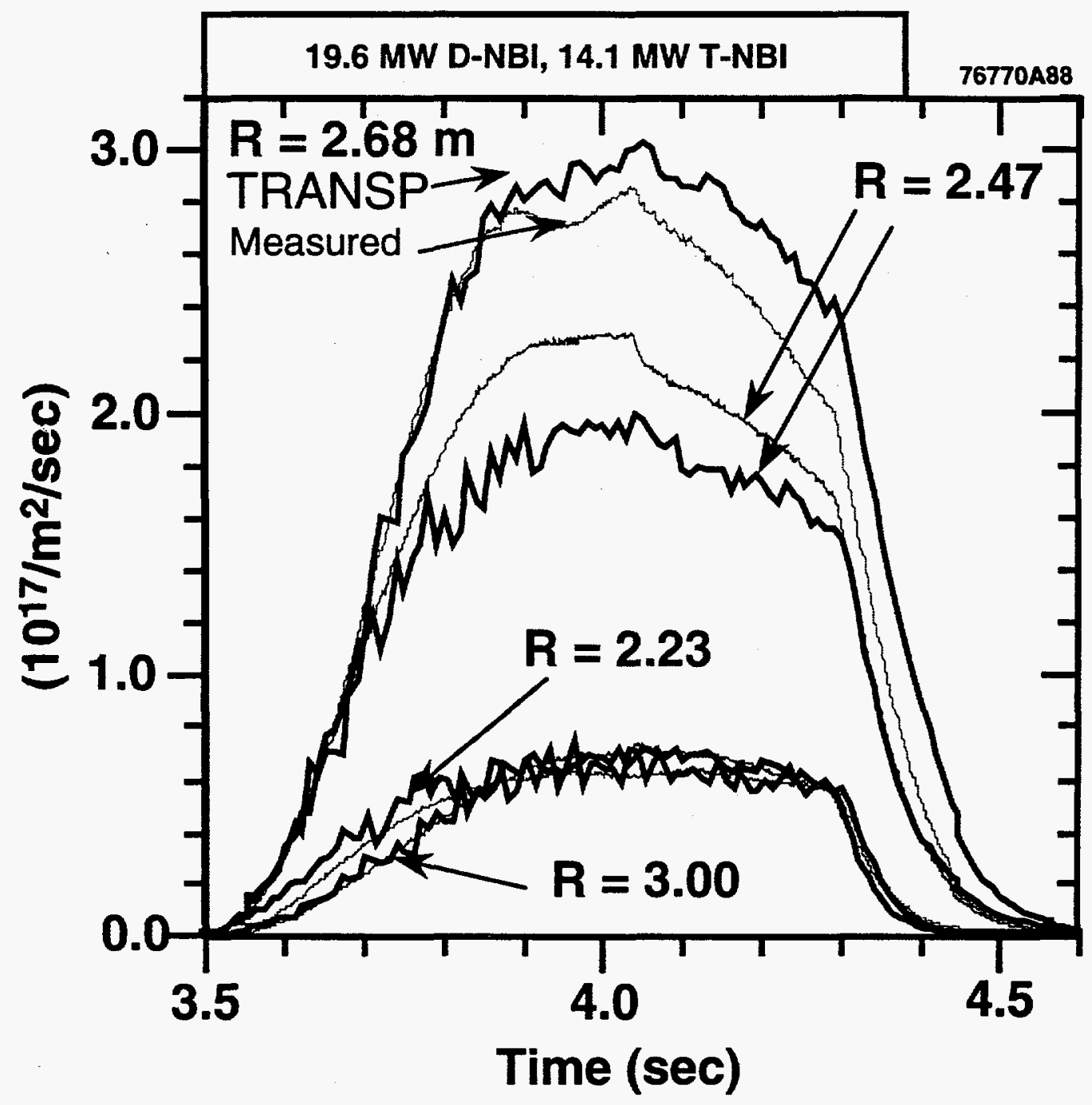

Fig. $2(b)$ 


\section{Chordal DT neutron emission profiles DT-NBI supershot}

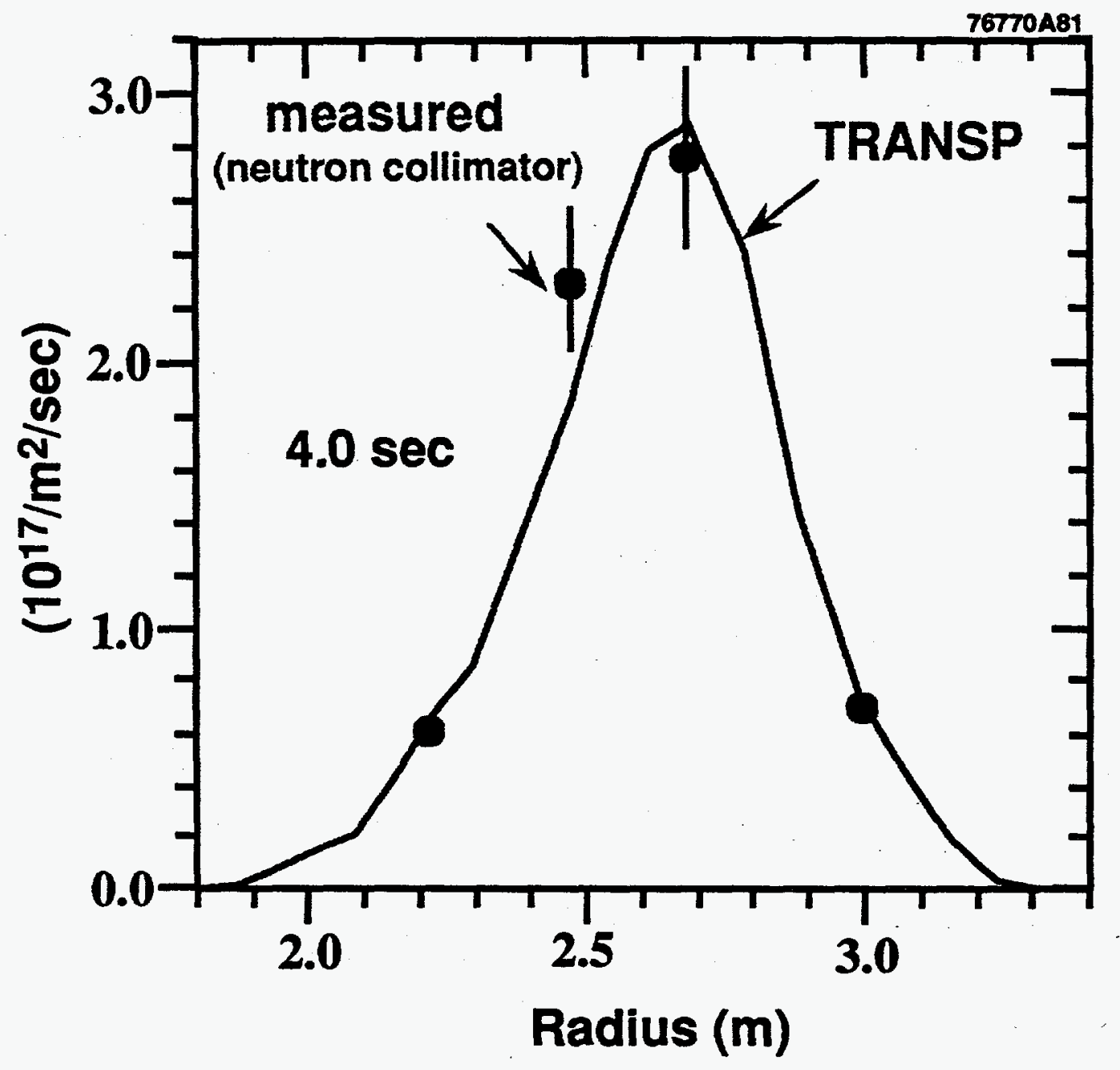

Fig. $2(c)$ 


\section{Central Fusion Power Assuming no fast ion mixing}

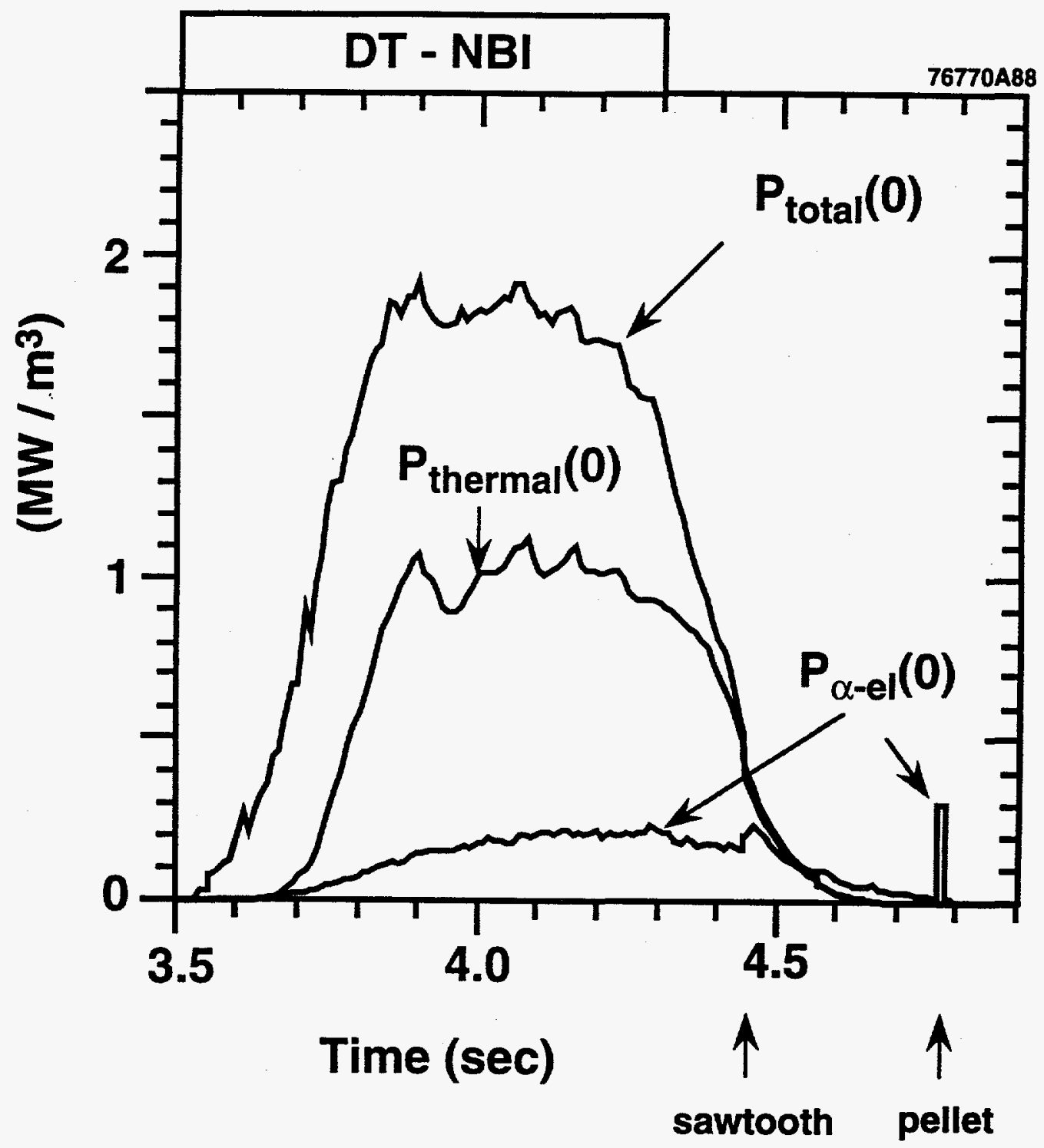

Fig. 3 
Profiles of fast ion slowing down times

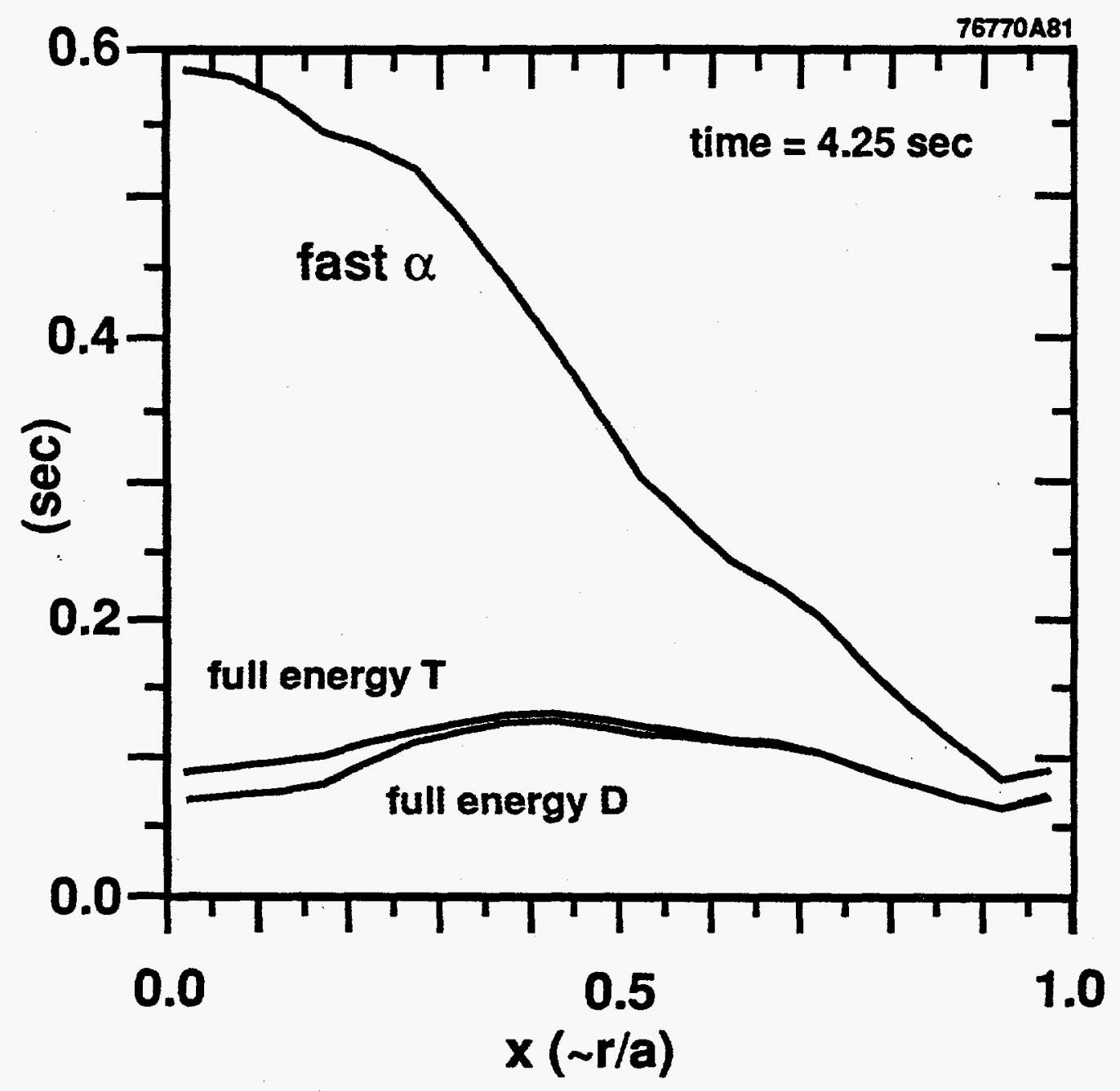

Fig. $4(a)$ 
Profiles of fast ion pitch angle scattering times

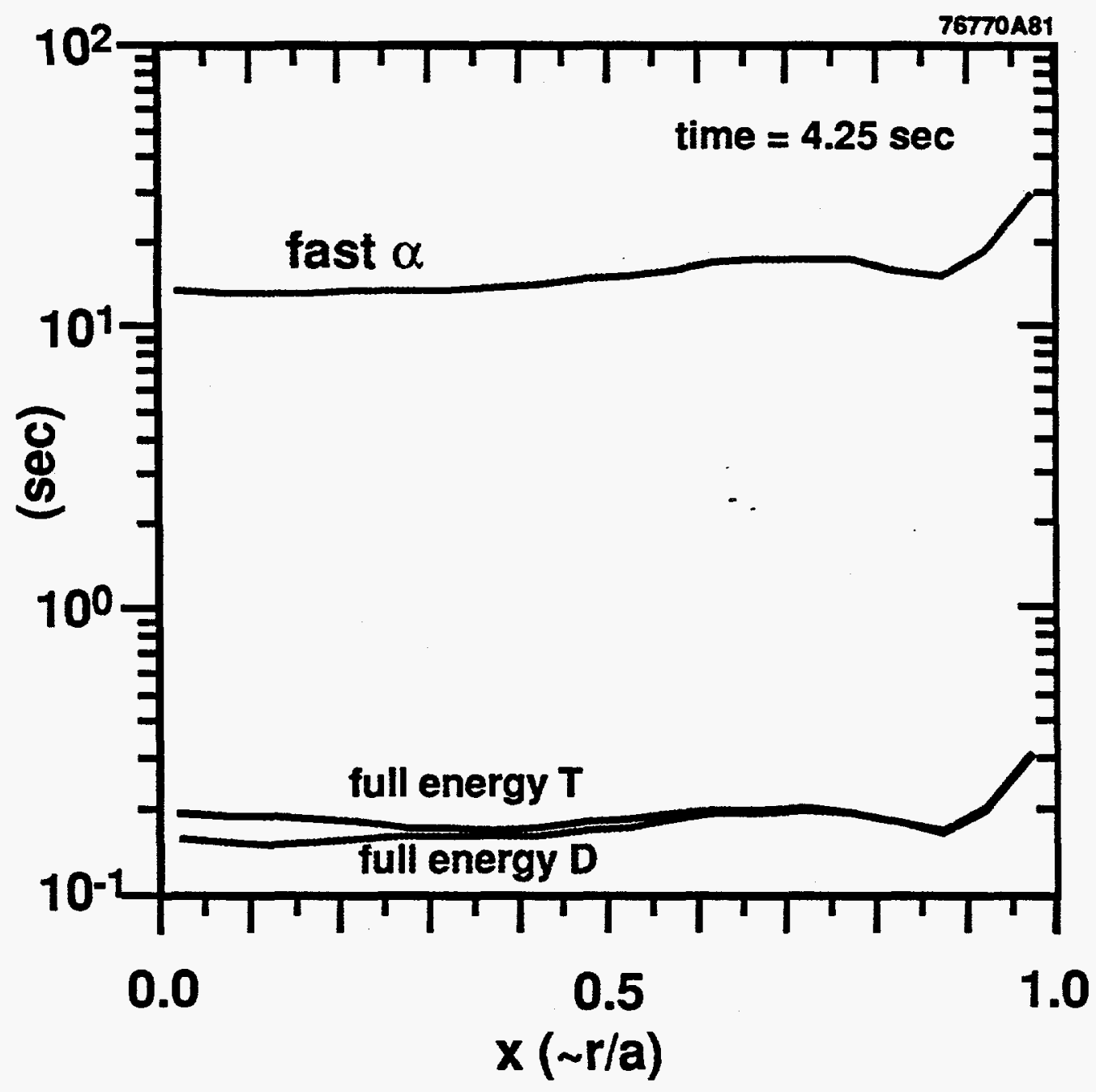

Fig. $4(b)$ 


\section{Electron heating profiles}

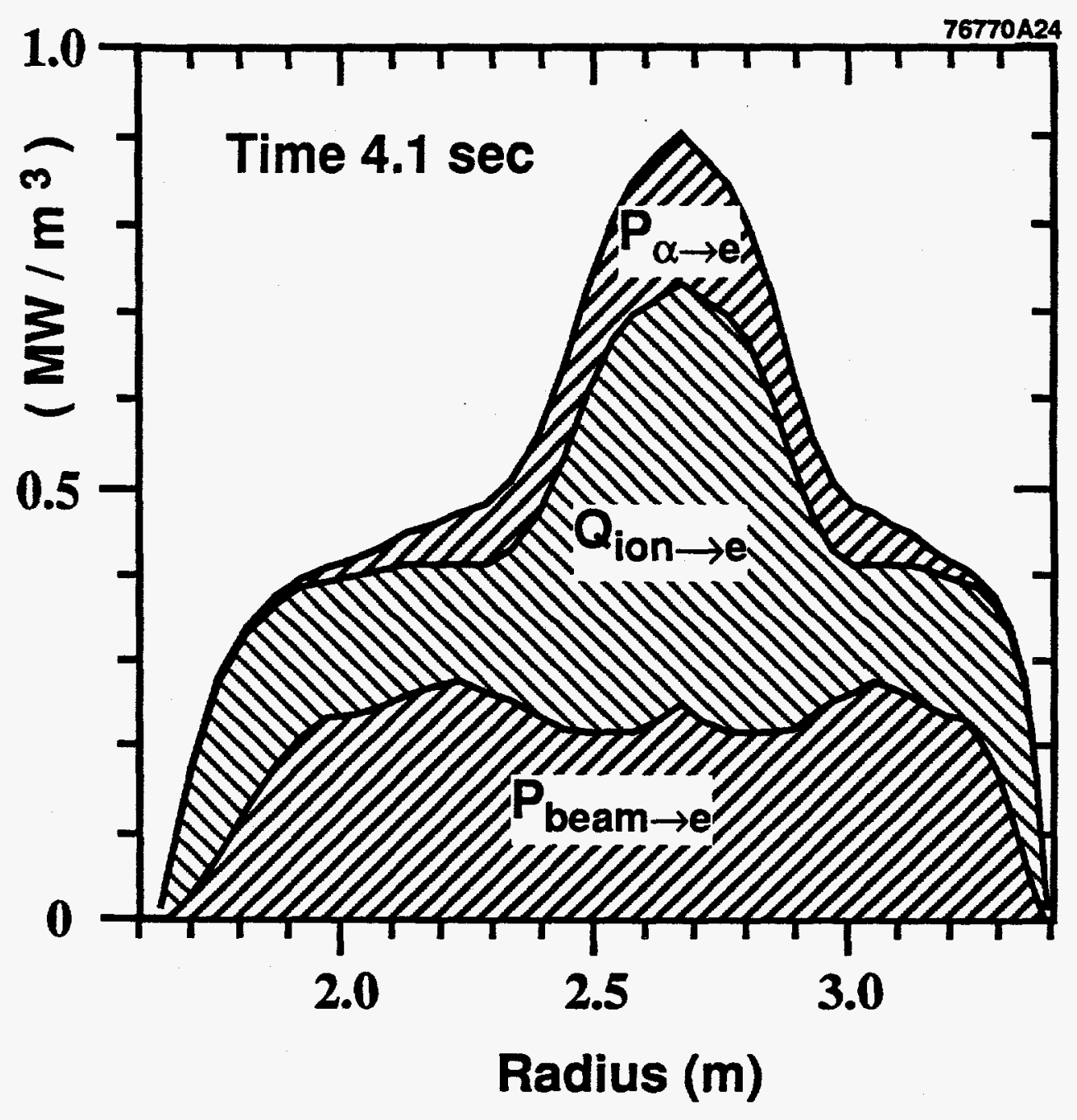

Fig. 5 


\section{Central alpha parameters}

With ripple, fast ion mixing

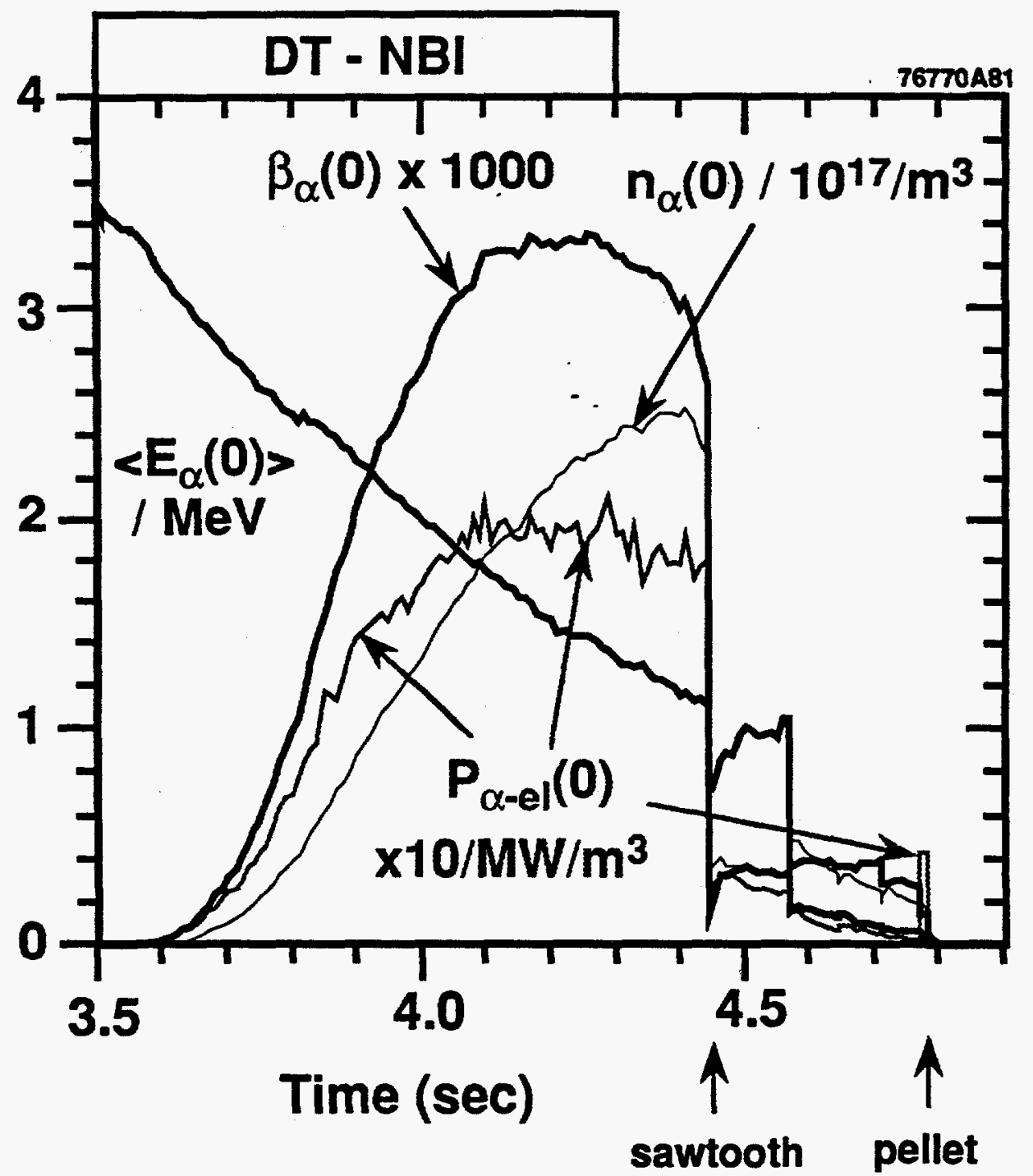

Fig. 6(a) 


\section{Central alpha parameters}

With ripple, no fast ion mixing

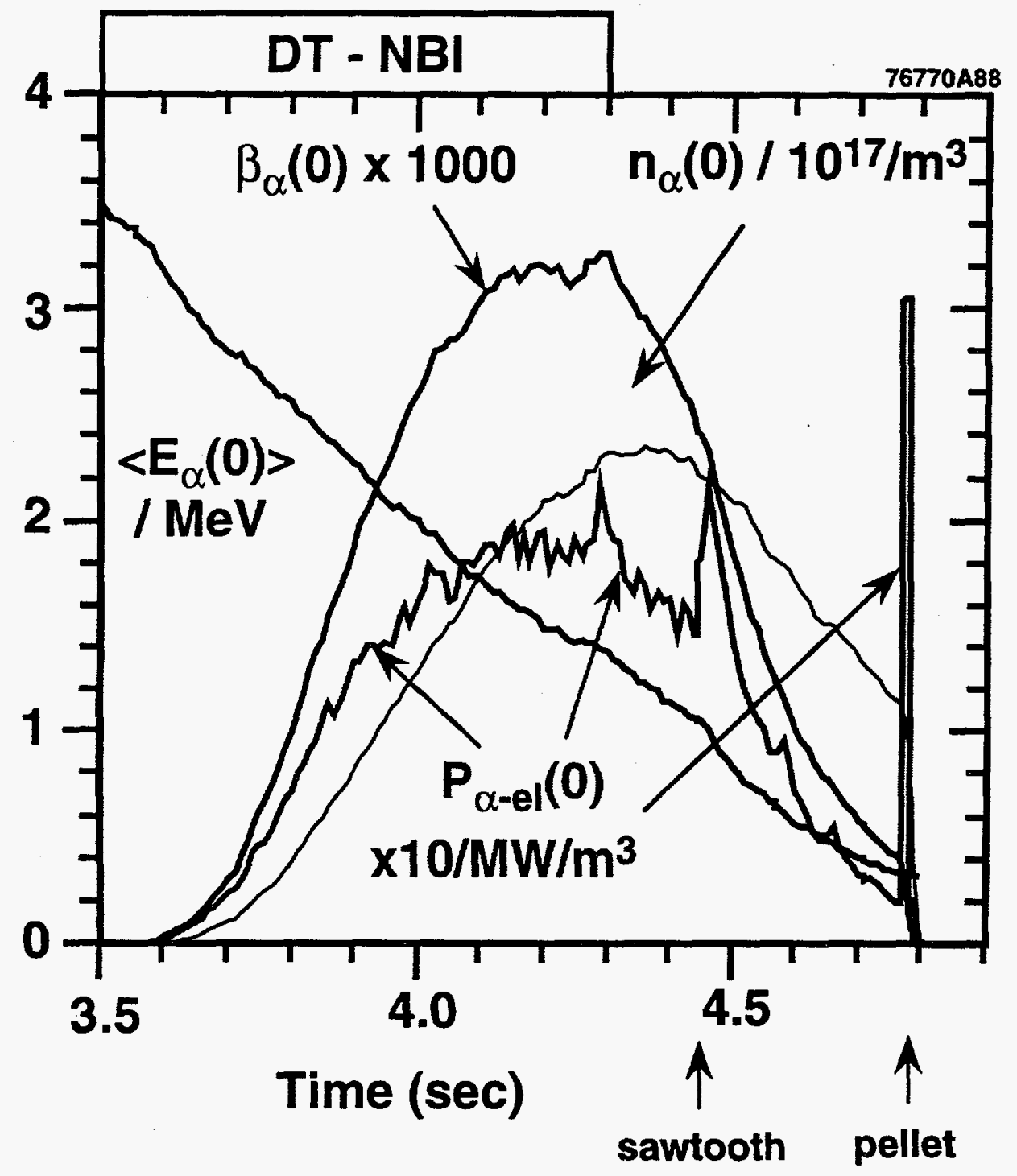

Fig. $6(b)$ 


\section{$\mathrm{n}_{\alpha}$ and $\mathrm{q}_{\psi}$ profiles}

\section{After sawtooth crash}

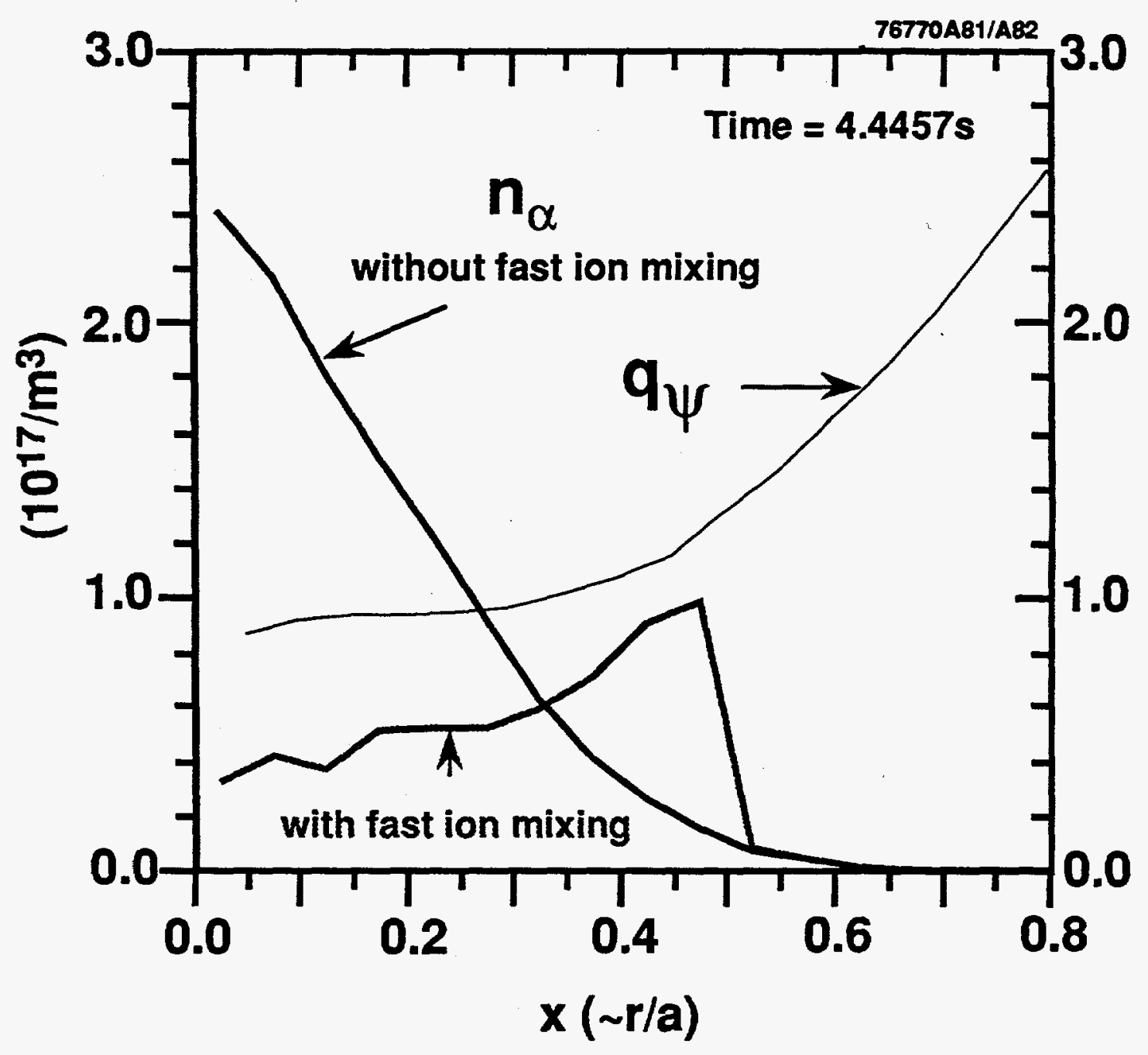

Fig. 7 
$\mathbf{n}_{\alpha}$ profiles

End of NBI

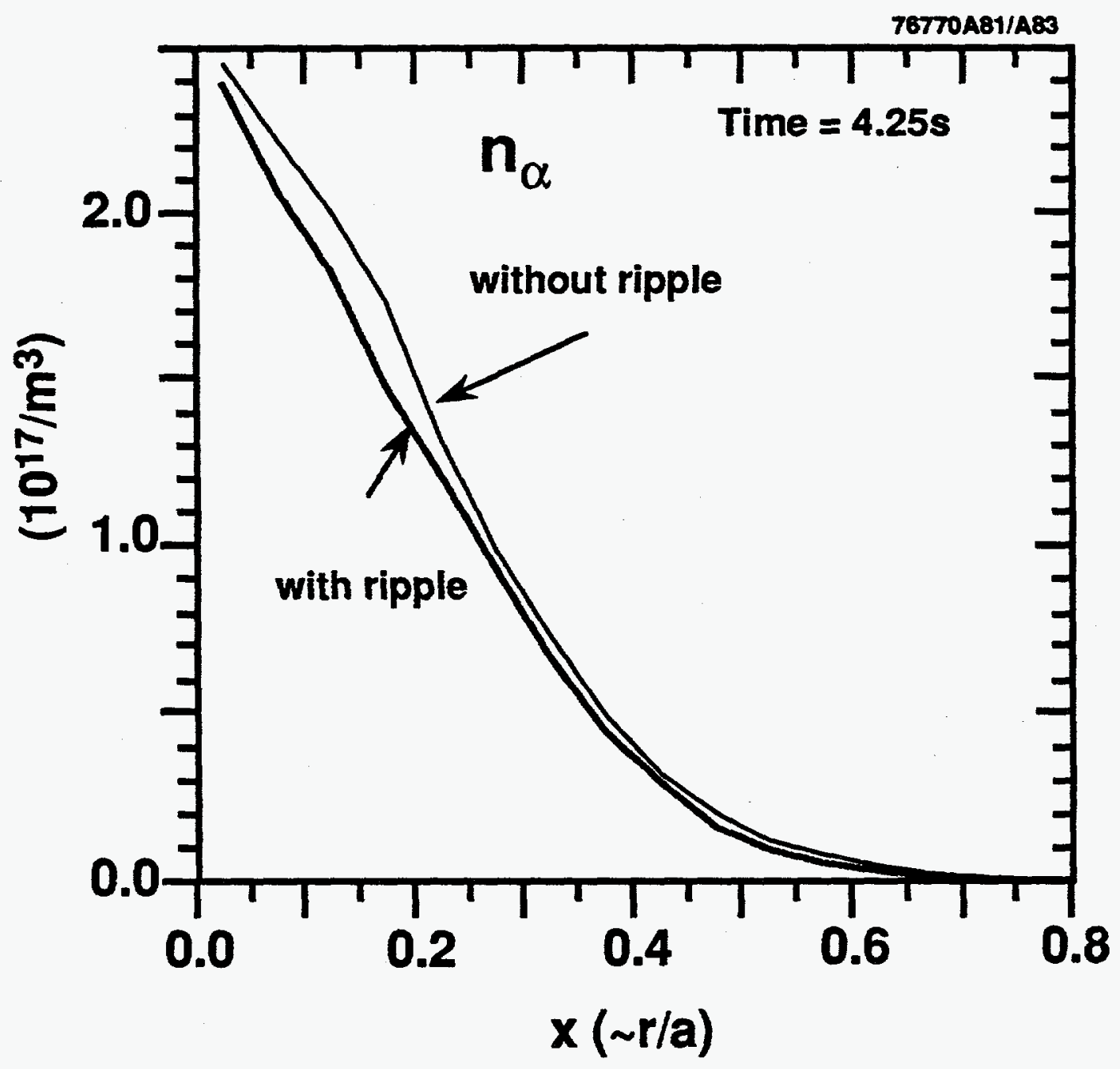

Fig. $8(a)$ 


\section{$\beta_{\alpha}$ profiles \\ End of NBI}

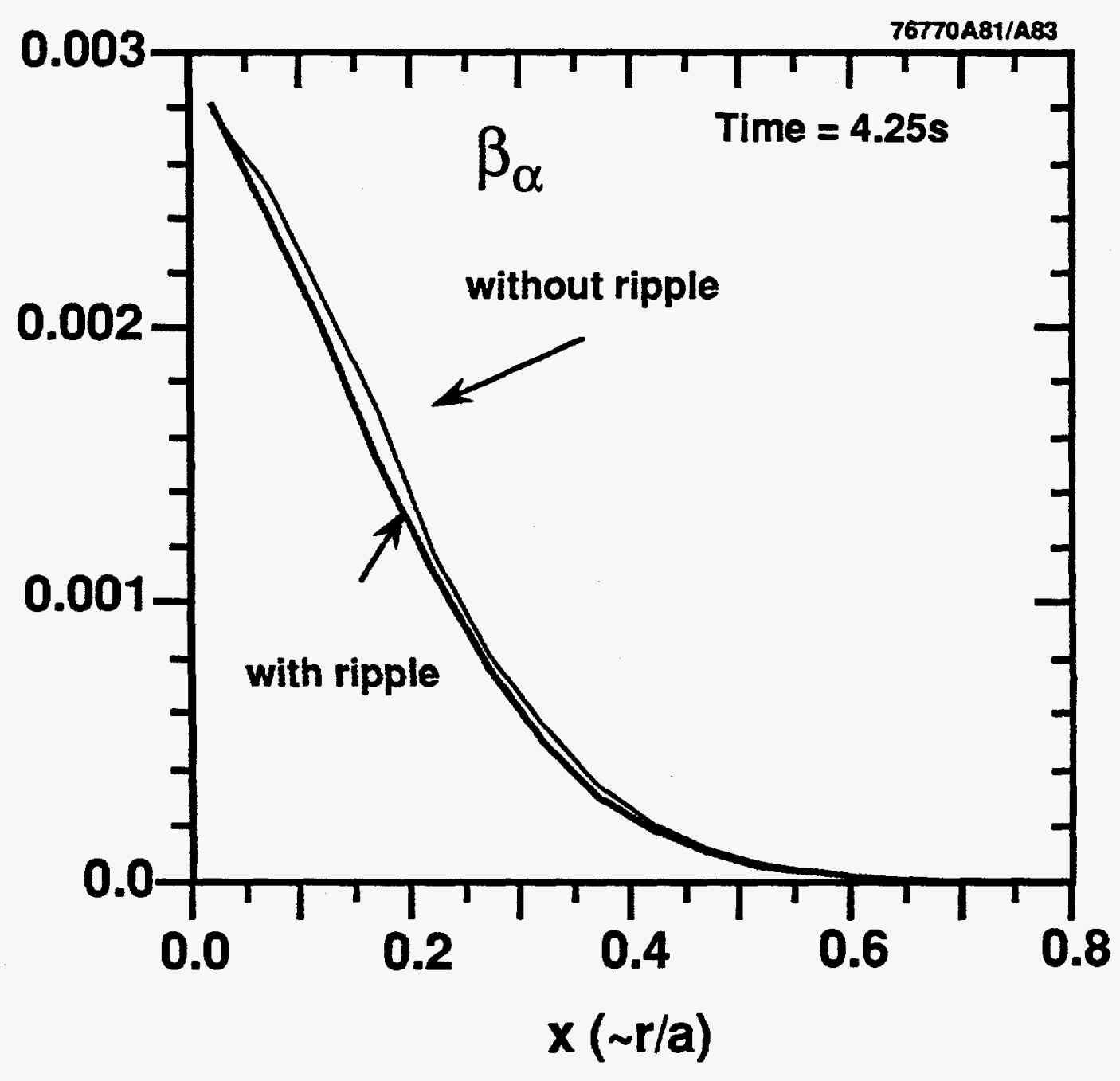

Fig. $8(b)$ 


\section{Magnetic Flux surfaces and zones for sampling distributions}

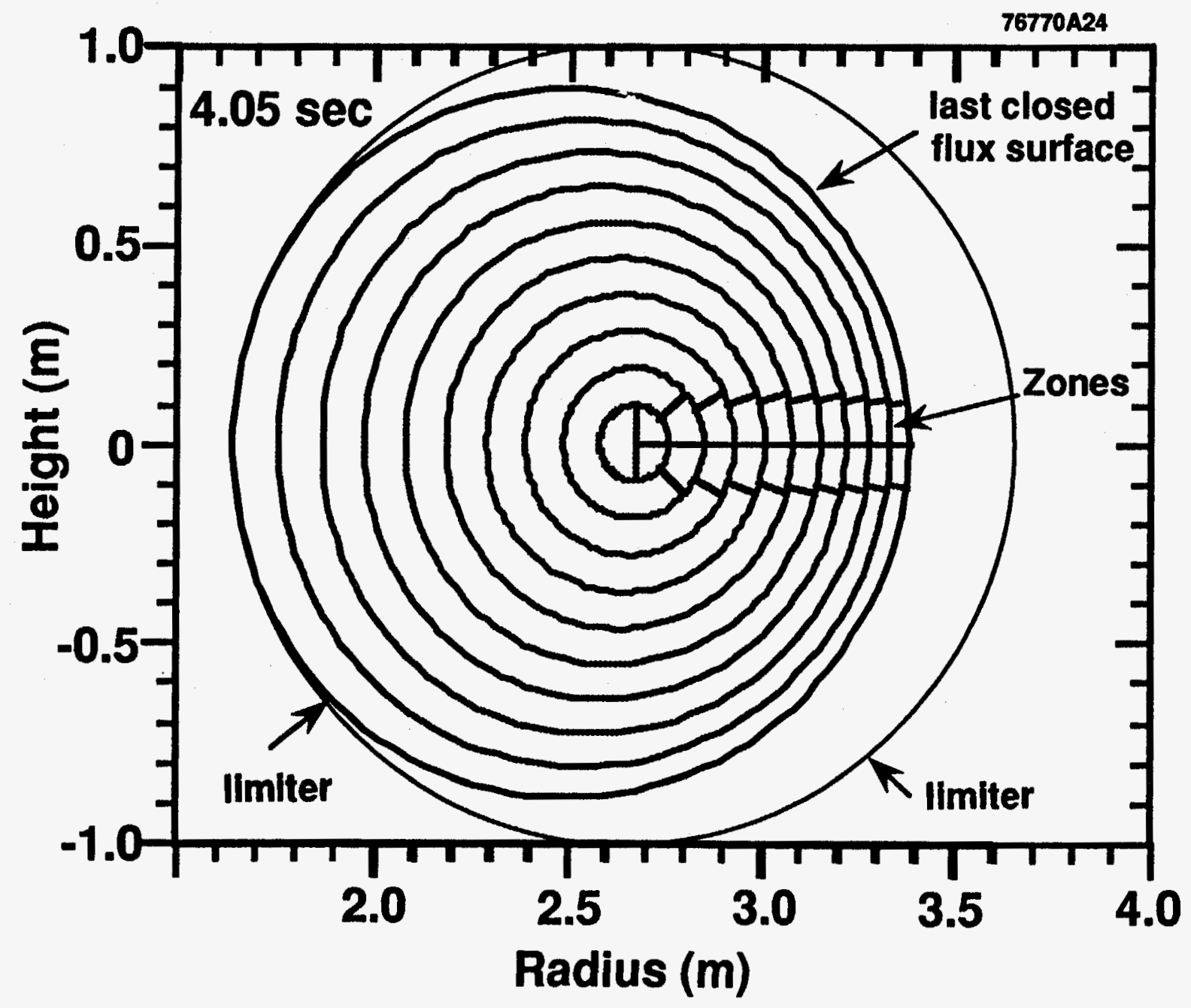




\section{Alpha distributions at $\mathrm{x}=0.5$ No ripple}

Time $=4.43 \mathrm{~s}$ (before sawtooth)

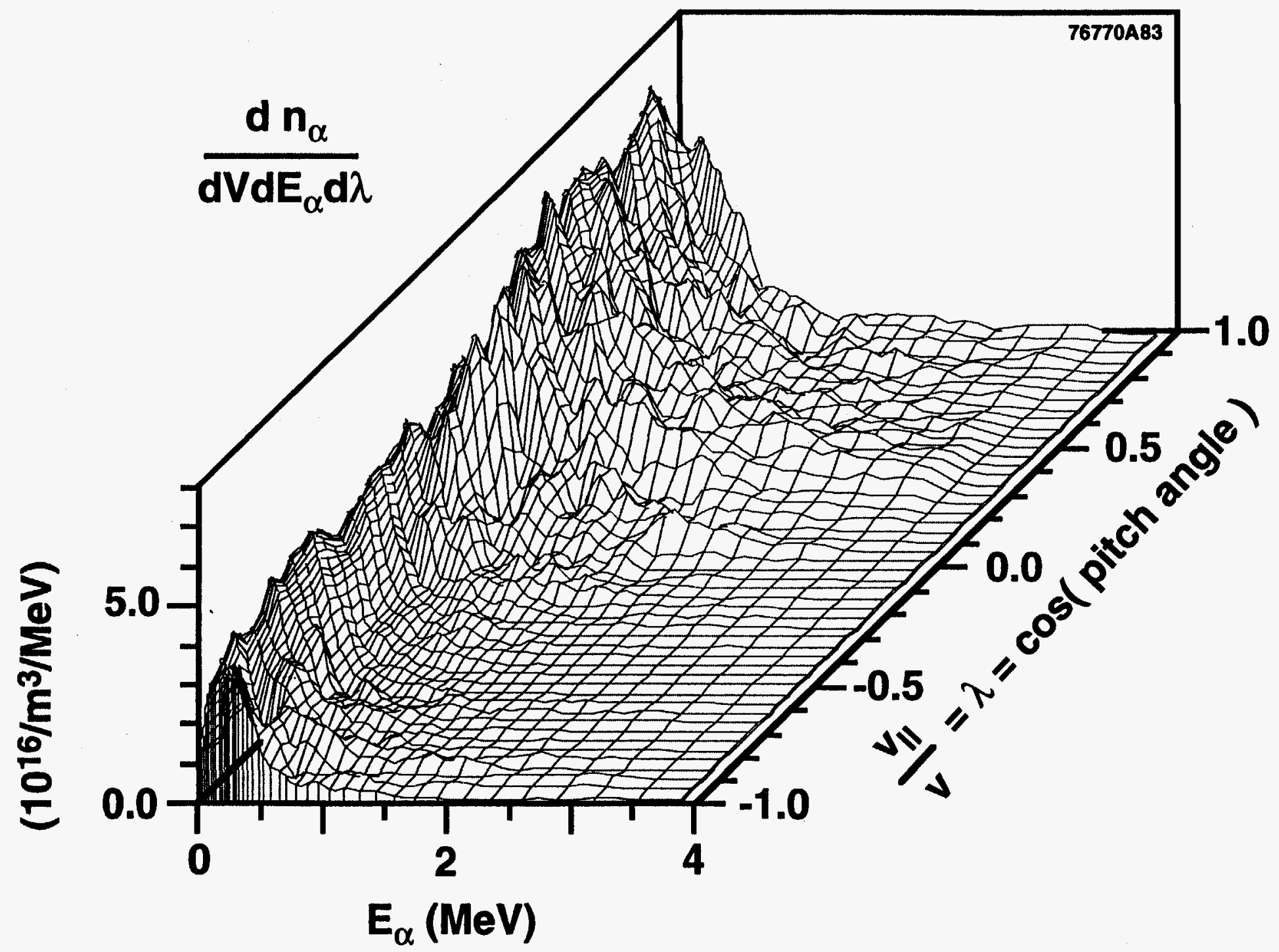




\section{Alpha distributions at $\mathrm{x}=0.5$ With ripple}

\section{Time $=4.43 \mathrm{~s}$ (before sawtooth)}

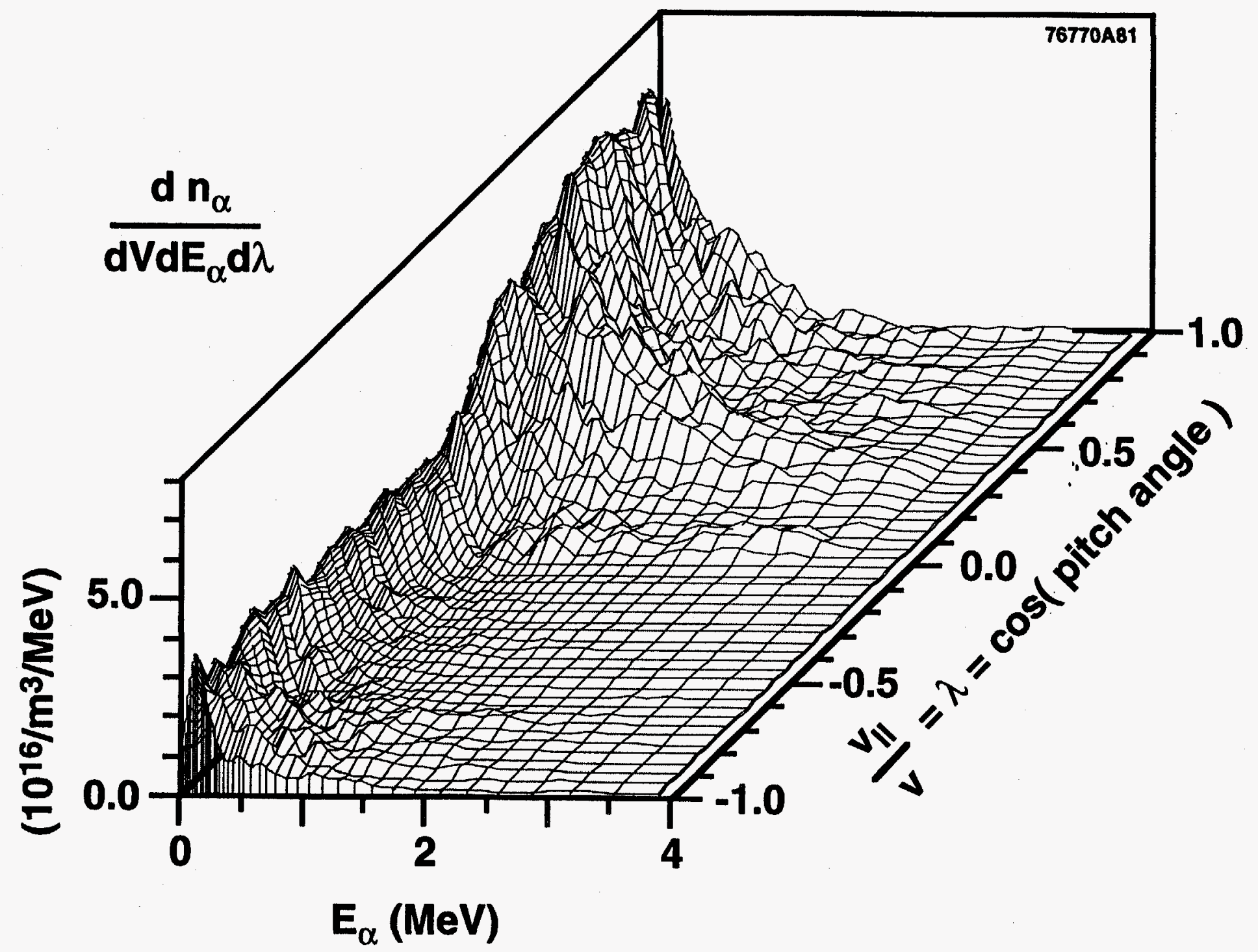




\section{Alpha distributions at $\mathrm{x}=\mathbf{0 . 7}$ No ripple}

Time $=4.43 \mathrm{~s}$ (before sawtooth)

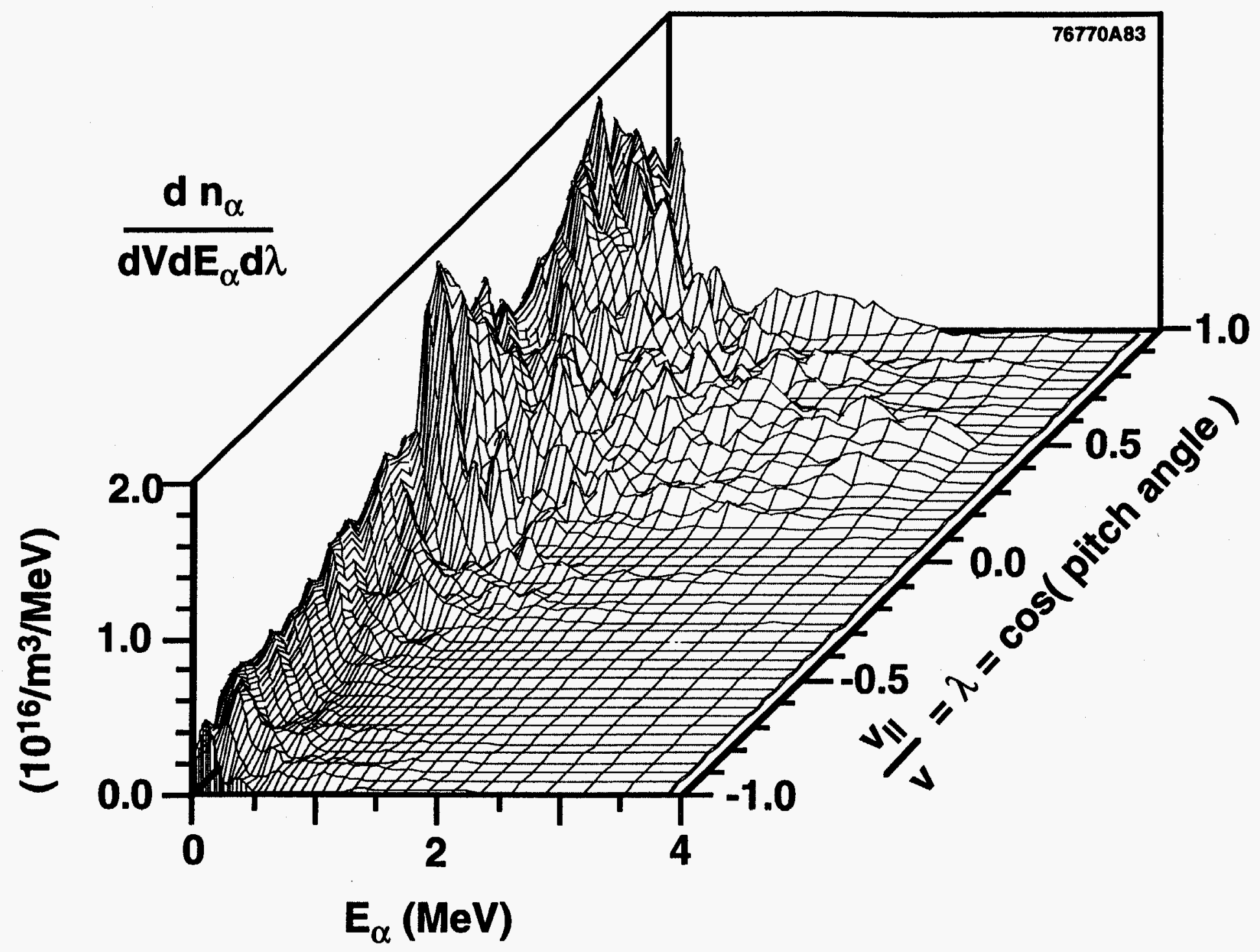




\section{Alpha distributions at $\mathrm{x}=\mathbf{0 . 7}$ With ripple}

Time $=4.43 \mathrm{~s}$ (before sawtooth)

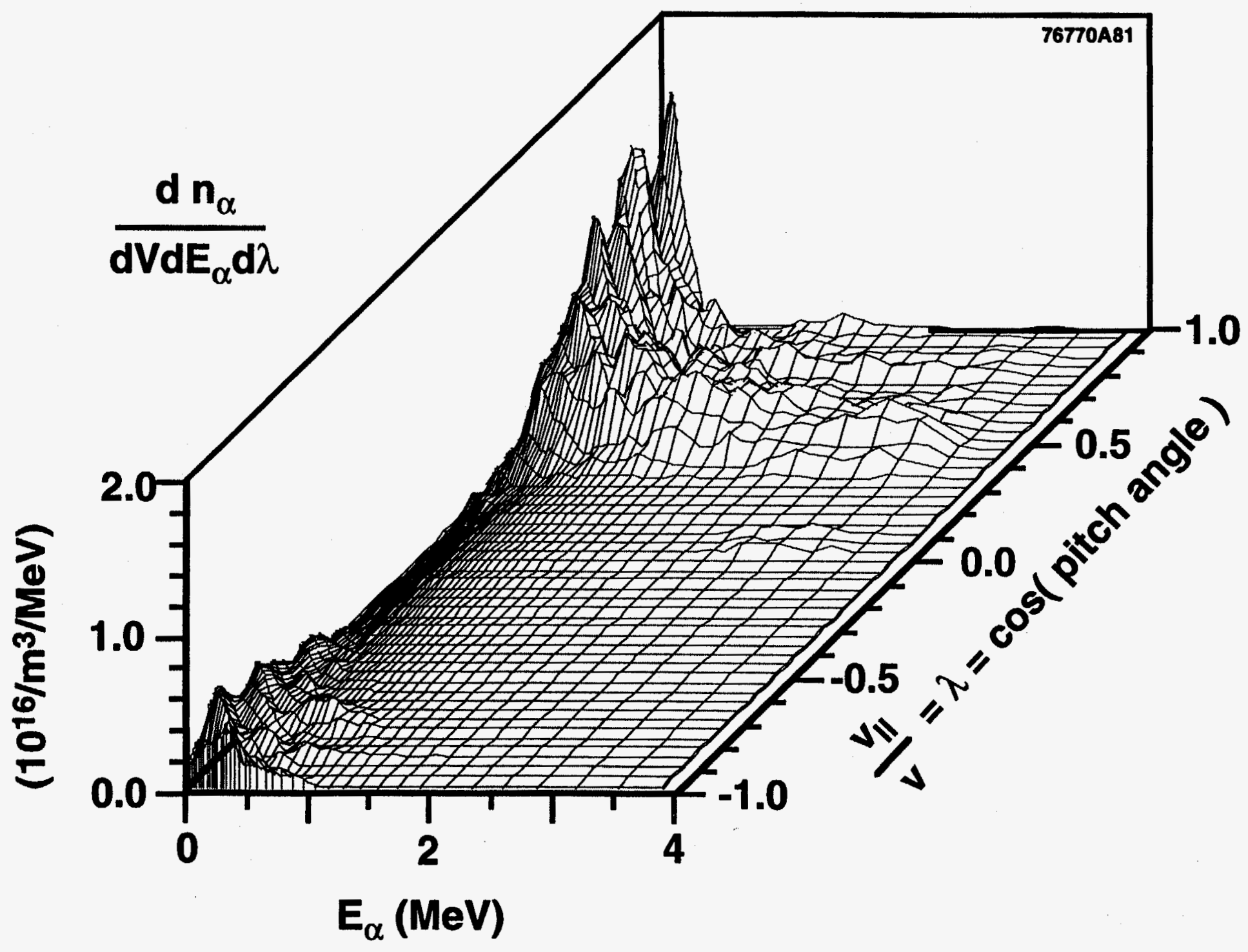




\section{Alpha density radial profiles}

$\lambda$ averaged from -0.1 to +0.1

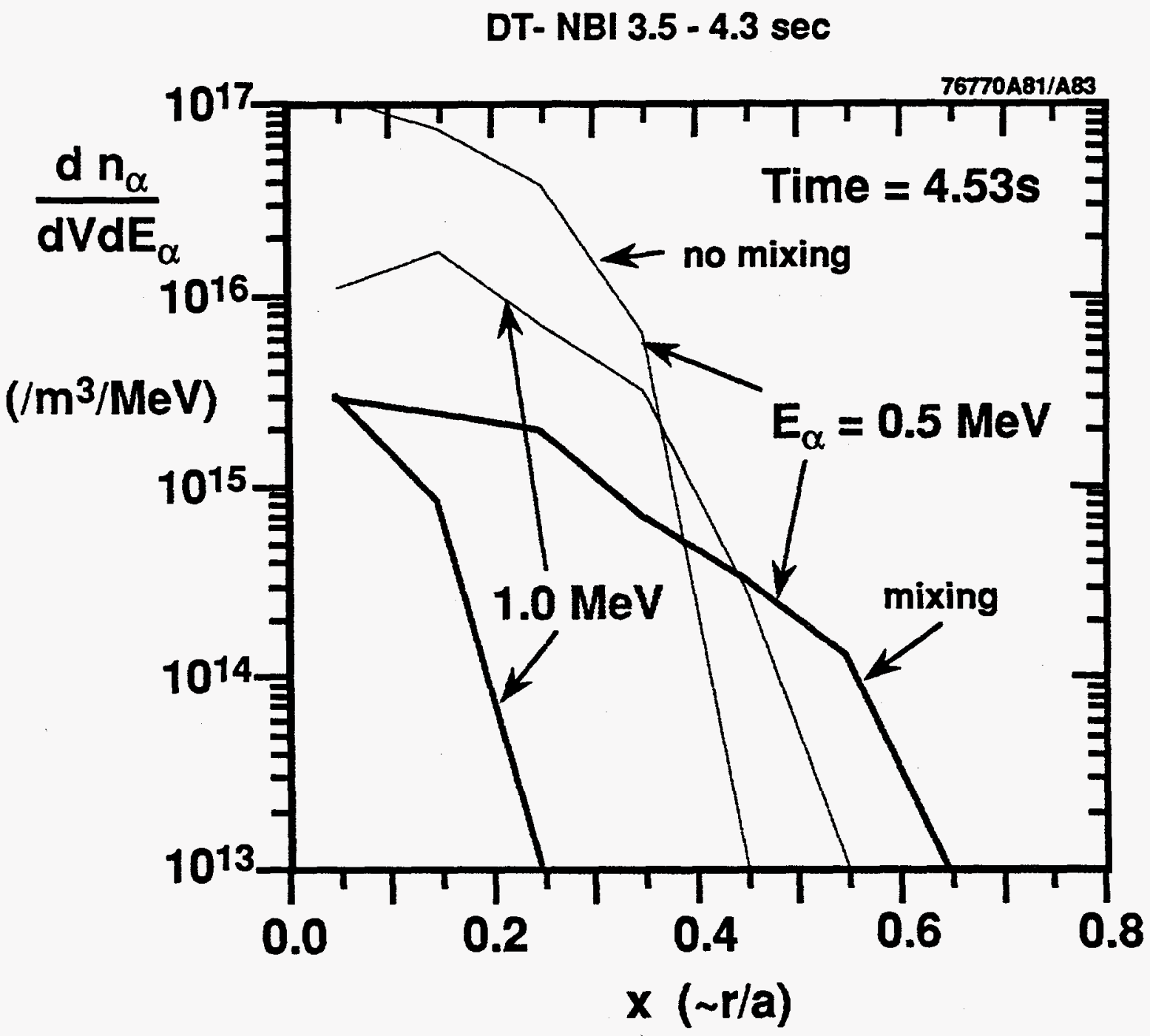

Fig. 12 


\section{Alpha pitch angle distributions \\ No ripple, no mixing}

$$
x=0.5 \text { Time }=4.53 \mathrm{sec}
$$

19.4 MW T-NBI, 14.0 MW D-NBI from 3.5 to $4.3 \mathrm{sec}$

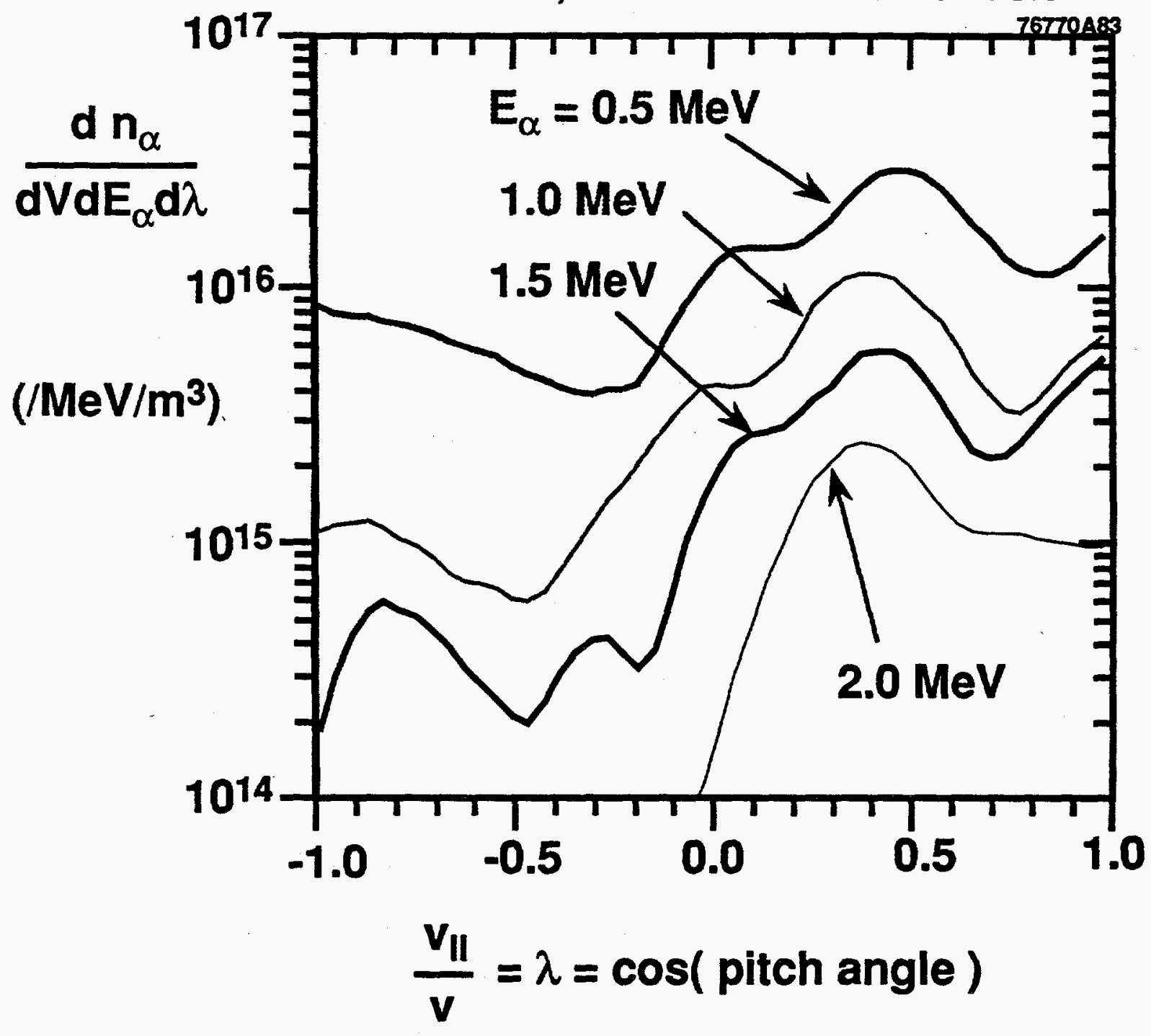




\section{Alpha pitch angle distributions \\ No ripple, with mixing}

$$
x=0.5 \quad \text { Time }=4.53 \mathrm{sec}
$$

19.4 MW T-NBI, 14.0 MW D-NBl from 3.5 to $4.3 \mathrm{sec}$

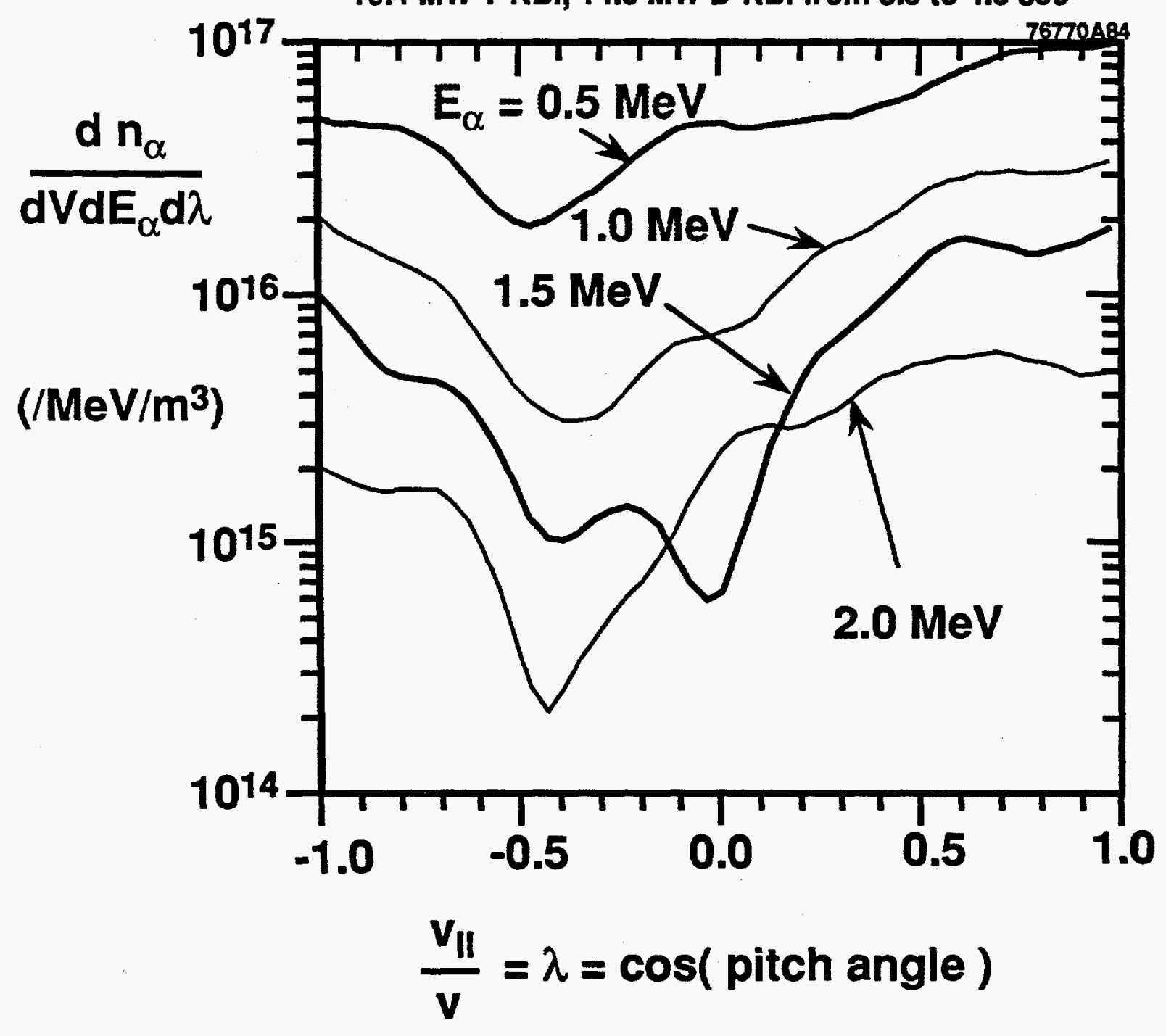

Fig. $13(b)$ 


\section{Alpha pitch angle distributions With ripple and mixing}

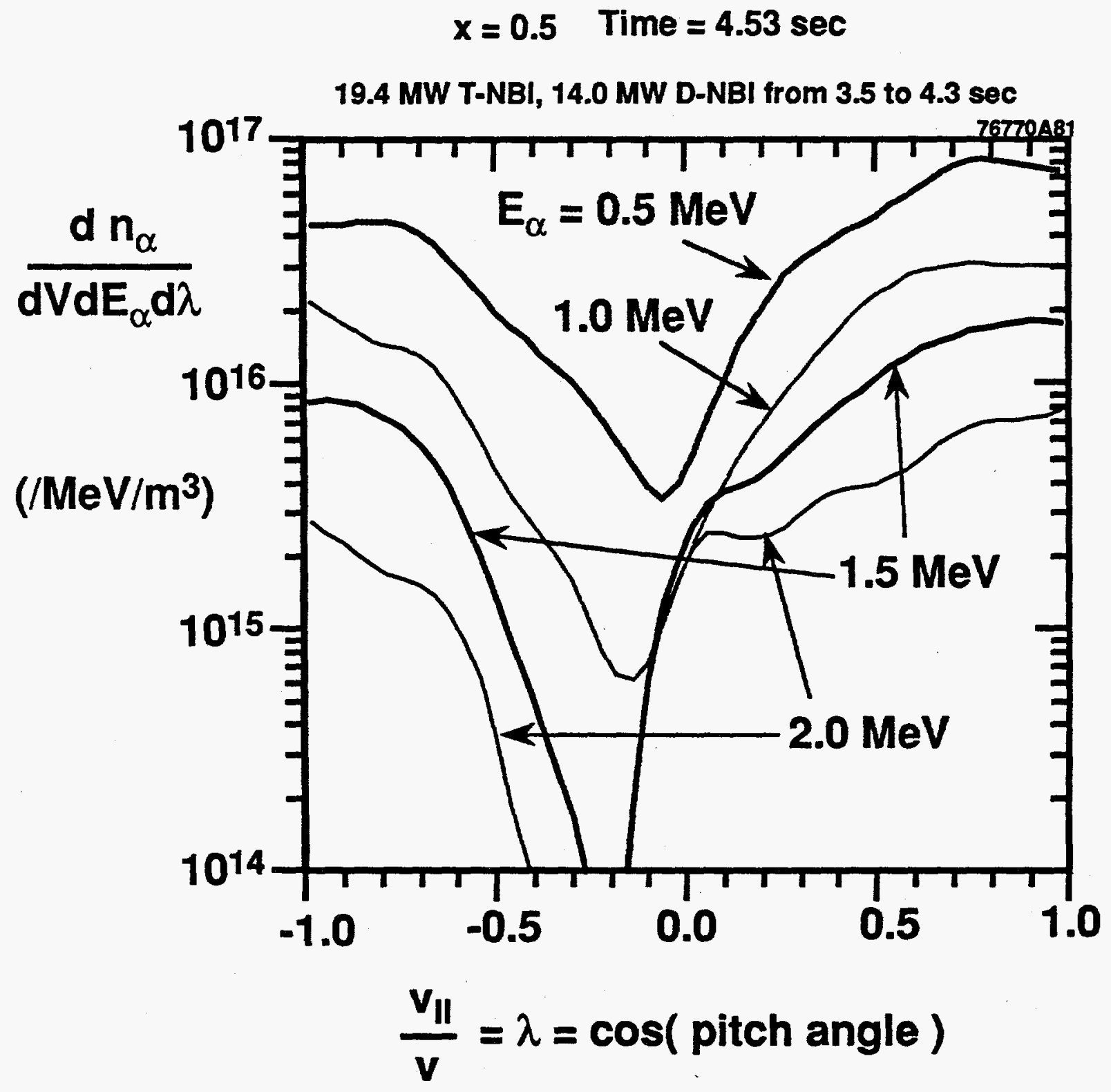

Fig. $13(\mathrm{c})$ 


\section{Central alpha energy distributions}

\section{DT Supershot with Fusion Yield = 7.5 MW}

19.4 MW T-NBI, 14.0 MW D-NBI from 3.5 to $4.3 \mathrm{sec}$

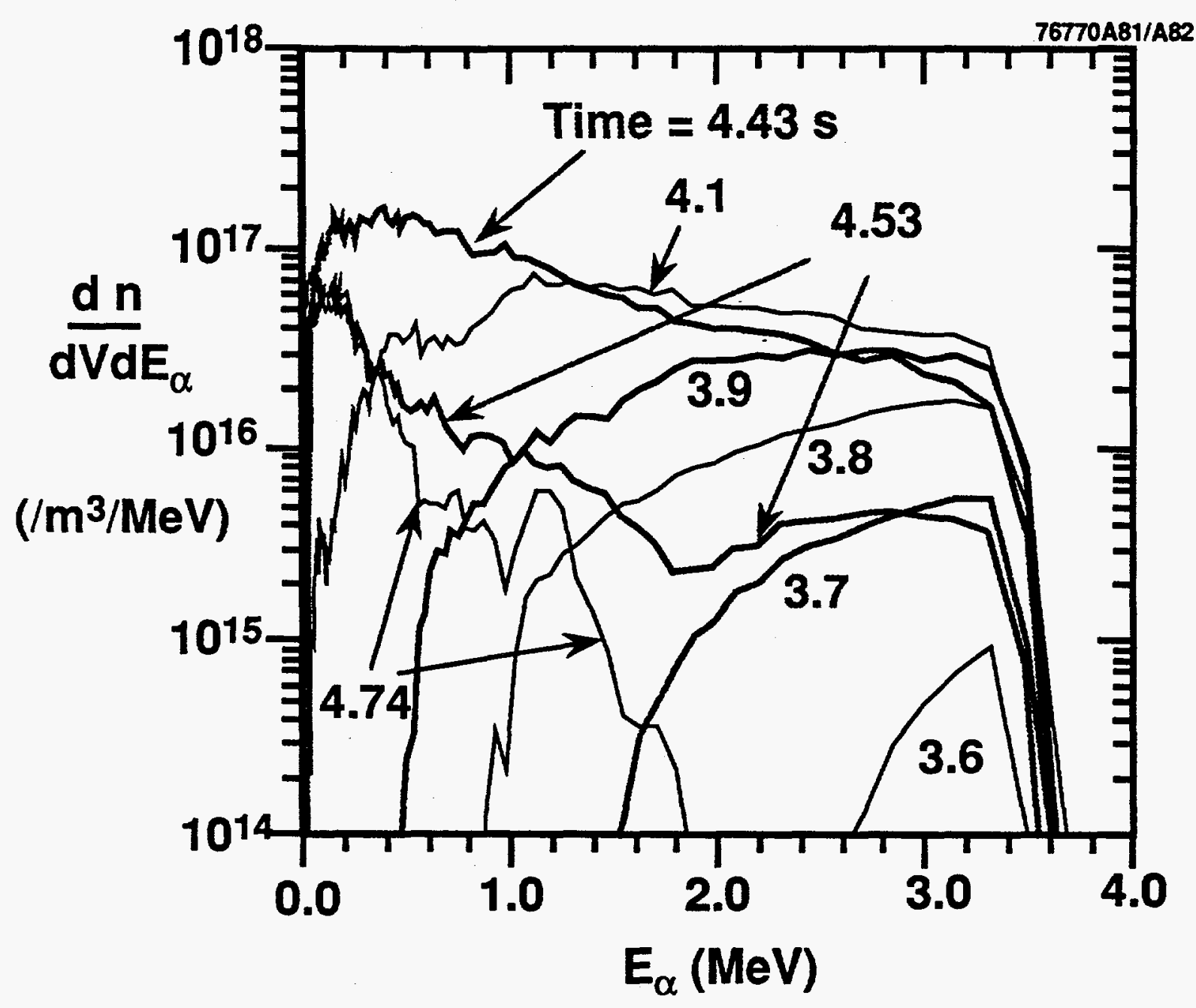

Fig. 14 
Dr. F. Paoloni, Univ, of Wollongong, AUSTRALIA

Prof. R.C. Cross, Univ. of Sydney, AUSTRALIA

Plasma Research Lab., Australian Nat. Univ., AUSTRALIA

Prof. I.R. Jones, Flinders Univ, AUSTRALIA

Prof. F. Cap, Inst. for Theoretical Physics, AUSTRIA

Prof. M. Heindler, Institut für Theoretische Physik, AUSTRIA

Prof. M. Goossens, Astronomisch Instituut, BELGIUM

Ecole Royale Militaire, Lab. de Phy. Plasmas, BELGIUM

Commission-European, DG. XII-Fusion Prog., BELGIUM

Prof. R. Bouciqué, Rijksuniversiteit Gent, BELGIUM

Dr. P.H. Sakanaka, Instituto Fisica, BRAZIL

Prof. Dr. I.C. Nascimento, Instituto Fisica, Sao Paulo, BRAZIL Instituto Nacional De Pesquisas Espaciais-INPE, BRAZIL Documents Office, Atomic Energy of Canada Lto., CANADA Ms. M. Morin, CCFMTokamak de Varennes, CANADA Dr. M.P. Bachynski, MPB Technologies, Inc., CANADA Dr. H.M. Skarsgard, Univ. of Saskatchewan, CANADA Prof. J. Teichmann, Univ. of Montreal, CANADA Prof. S.R. Sreonivasan, Univ. of Calgary, CANADA Prof. R. Marchand, INRS-Energie et Materiaux, CANADA Dr. R. Bolton, Centre canadien de fusion magnétique, CANADA Dr. C.R. James, Univ. of Alberta, CANADA Dr. P. Lukác, Komenského Universzita, CZECHO-SLOVAKIA The Librarian, Culham Laboratory, ENGLAND Library, R61, Rutherford Appleton Laboratory, ENGLAND Mrs. S.A. Hutchinson, JET Library, ENGLAND Dr. S.C. Sharma, Univ. of South Pacific, FIJ!I ISLANDS

P. Mähönen, Univ. of Helsinki, FINLAND

Prof. M.N. Bussac, Ecole Polytechnique, FRANCE C. Mouttet, Lab. de Physique des Milieux lonisés, FRANCE J. Radet, CEN/CADARACHE - Bat 506, FRANCE Prof. E. Economou, Univ. of Crete, GREECE Ms. C. Rinni, Univ. of loannina, GREECE Preprint Libray, Hungarian Academy of Sci., HUNGARY Dr. B. DasGupta, Saha Inst of Nuclear Physics, INDIA Dr. P. Kaw, Inst. for Plasma Research, INDIA Dr. P. Rosenau, Israel Inst. of Technology, ISRAEL Librarian, Intemational Center for Theo Physics, ITALY Miss C. De Palo, Associazione EURATOM-ENEA , ITALY Dr. G. Grosso, Istituto di Fisica del Plasma, ITALY Prof. G. Rostangni, Istituto Gas lonizzati Del Cnr, ITALY
Dr. H. Yamato, Toshiba Res \& Devel Center, JAPAN

Prof. I. Kawakami, Hiroshima Univ., JAPAN

Prof. K Nishikawa, Hiroshima Univ., JAPAN

Librarian, Naka Fusion Research Establishment, JAERI, JAPAN

Director, Japan Atomic Energy Research Inst., JAPAN

Prof. S. Itoh, Kyushu Univ., JAPAN

Research Info. Ctr., National Instit for Fusion Science, JAPAN

Prof. S. Tanaka, Kyoto Univ., JAPAN

Library, Kyoto Univ., JAPAN

Prof. N. Inoue, Univ. of Tokyo, JAPAN

Secretary, Plasma Section, Electrotechnical Lab., JAPAN

Dr. O. Mitarai, Kumamoto Inst. of Technology, JAPAN

Dr. G.S. Lee, Korea Basic Sci. Ctr., KOREA

J. Hyeon-Sook, Korea Atomic Energy Research Inst, KOREA D.I. Choi, The Korea Adv. Inst. of Sci. \& Tech., KOREA Leandro Melendez Lugo, Inst. Nac1. de Inves. Nuct, MEXICO Prof. B.S. Liley, Univ. of Waikato, NEW ZEALAND Inst of Physics, Chinese Acad Sci PEOPLE'S REP. OF CHINA Library, Inst. of Plasma Physics, PEOPLE'S REP. OF CHINA Tśinghua Univ. Library, PEOPLE'S REPUBLIC OF CHINA Z. Li, S.W. Inst Physics, PEOPLE'S REPUBLIC OF CHINA Prof. J.A.C. Cabral, Instituto Superior Tecnico, PORTUGAL Prof. M.A. Hellberg, Univ. of Natal, S. AFRICA

Prof. D.E. Kim, Pohang inst. of Sci. \& Tech., SO. KOREA

Prof. C.I.E.M.A.T, Fusion Division Library, SPAIN

Dr. L. Stenflo, Univ, of UMEA, SWEDEN

Library, Royal Inst. of Technology, SWEDEN

Prof. H. Wilhelmson, Chalmers Univ. of Tech., SWEDEN Centre Phys. Des Plasmas, Ecole Polytech, SWITZERLAND Bibliotheek, Inst. Voor Plasma-Fysica, THE NETHERLANDS Asst. Prof. Dr. S. Cakir, Middle East Tech. Univ., TURKEY Dr. V.A. Glukhikh,Sci. Res. Inst. Electrophys.I Apparatus, USSR Dr. D.D. Ryutov, Siberian Branch of Academy of Sa., USSR Dr. G.A. Eliseev, I.V. Kurchatov Inst, USSR Librarian, The Ukr.SSR Academy of Sciences, USSR Dr. L.M. Kovrizhnykh, Inst. of General Physics, USSR Kemforschungsanlage GmbH, Zentralbibliothek, W. GERMANY Bibliothek, Inst. Für Plasmaiorschung, W. GERMANY Prof. K Schindler, Ruhr-Universitát Bochum, W. GERMANY Dr. F. Wagner, (ASDEX), Max-Planck-Institut, W. GERMANY Librarian, Max-Planck-Institut, W. GERMANY 